\title{
4. PLANKTONIC FORAMINIFER FAUNAL VARIATIONS IN THE NORTHEASTERN INDIAN OCEAN: A HIGH-RESOLUTION RECORD OF THE PAST 800,000 YEARS FROM SITE $758^{1}$
}

\author{
Min-Te Chen ${ }^{2}$ and John Farrell ${ }^{2}$
}

\begin{abstract}
We present a high-resolution ( $6 \mathrm{k} . \mathrm{y}$. sample interval) record of planktonic foraminifer faunal variations for the past $800 \mathrm{k} . \mathrm{y}$. from ODP Site 758 in the northeastern Indian Ocean. The record is examined within the context of a coarse fraction stratigraphy which is a lithologic index of $\mathrm{CaCO}_{3}$ preservation, and an oxygen isotope stratigraphy which provides a chronostratigraphy and a record of climate change. Variations in the relative abundance of 27 planktonic foraminifer species primarily reflect fluctuations in the intensity of $\mathrm{CaCO}_{3}$ dissolution. $\mathrm{CaCO}_{3}$ dissolution covaries with climate fluctuations at a cyclicity of about $100 \mathrm{k} . \mathrm{y}$. Glacial-aged sediments are generally well preserved, as indicated by faunal and lithologic indices. Interglacial-aged sediments show poorer preservation. The $\sim 100-k . y$. cycles are superimposed upon the long-term Brunhes Dissolution Cycle. This cycle is characterized by an interval of poor preservation centered between 400 and $550 \mathrm{ka}$ and is bounded by good preservation events at 25 and $750 \mathrm{ka}$. Ecological factors also control variations in the foraminifer fauna. Changes in ecology are inferred from downcore fluctuations in the relative abundances of foraminifer species that have a similar level of resistance to dissolution. We focus on three species (Neogloboquadrina dutertrei, Pulleniatina obliquiloculata, Globorotalia menardii) with a relatively high resistance to dissolution. The long-term increase in $N$. dutertrei since at least $800 \mathrm{ka}$ is interpreted as either a gradual decrease in the sea-surface salinity, or an increase in the biogenic productivity of the surface waters. These factors may be controlled by the strength of the monsoon climate. Extremely high abundances of $P$. obliquiloculata are observed in certain downcore intervals and are interpreted as times when the surface water conditions in the northeast Indian Ocean were similar to those in the modern western tropical Pacific, but not the modern Indian Ocean.
\end{abstract}

\section{INTRODUCTION}

A major objective of Ocean Drilling Program (ODP) Leg 121 is the investigation of Neogene climate and ocean history in the Indian Ocean. A significant portion of this history is recorded in planktonic foraminifers. Climate and ocean change is recorded by variations in faunal composition, by fluctuations in the chemical composition of foraminifer skeletons, and by the preservation state of foraminifers. With faunal information, we can evaluate the ecological response to climatically-induced changes in surface water conditions. Faunal assemblages also reflect the preservation state of $\mathrm{CaCO}_{3}$ on the seafloor which documents fluctuations in the intensity of $\mathrm{CaCO}_{3}$ dissolution, and thus changes in abyssal water chemistry.

The sedimentary section recovered at ODP Site 758 , located in the northeastern Indian Ocean (Fig. 1), provides an excellent opportunity to study oceanic and climatic change. The Quaternary section was recovered in multiple advanced piston cores (APC) as a continuous and undisturbed sequence which accumulated at an average rate of $1.6 \mathrm{~cm} / \mathrm{k} . y$. (Farrell and Janecek, this volume). The specific objectives of this study are to: (1) document variations in the relative abundance of planktonic foraminifer species in late Quaternary sediments $(0-800 \mathrm{ka}) ;(2)$ determine the timing and magnitude of faunal change; and (3) identify the ecological and preservational processes which affected the assemblages.

\section{REGIONAL SETTING}

Site 758 was drilled at $5^{\circ} 23.05^{\prime} \mathrm{N}, 90^{\circ} 21.67^{\prime} \mathrm{E}$ in a water depth of $2924 \mathrm{~m}$ (Fig. 1). The site is located at the southernmost end of the Bay of Bengal, atop the Ninetyeast Ridge, at least $1000 \mathrm{~m}$

\footnotetext{
${ }^{1}$ Weissel, J., Peirce, J., Taylor, E., Alt, J., et al., 1991. Proc. ODP, Sci. Results, 121: College Station, TX (Ocean Drilling Program).

${ }^{2}$ Department of Geological Sciences, Brown University, Providence, RI 02912 1846, U.S.A.
}

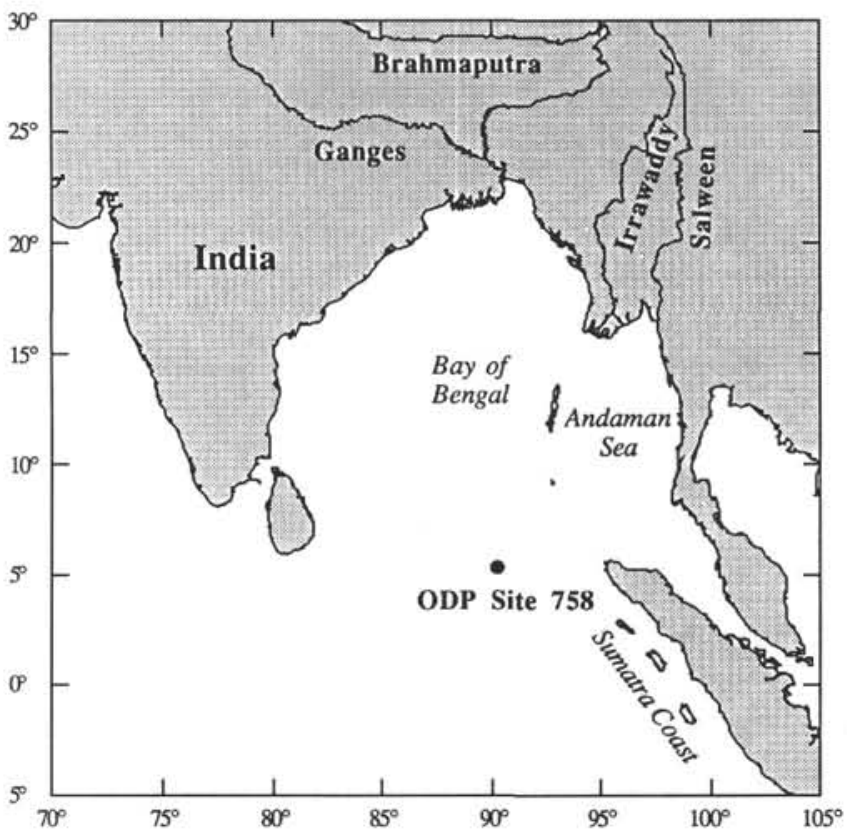

Figure 1. Location of ODP Site 758 in the northeastern Indian Ocean.

above the Bengal Fan. This fan is the world's largest, and it is built of terrigenous sediments carried from the Himalayan region by the Ganges-Brahmaputra, Irrawaddy, and Salween river systems to the Bay of Bengal (Curray and Moore, 1971; Goldberg and Griffin, 1970). The Quaternary sediments at Site 758 are predominantly biogenic calcareous ooze with secondary amounts of terrigenous clay and occasional intercalated volcanic ash layers (Shipboard Scientific Party, 1989). 
The oceanography and climatology of the North Indian Ocean are strongly controlled by the monsoon climate regime which is characterized by the seasonal reversal of wind and surface water circulation. In the northern Bay of Bengal, the surface waters undergo dramatic changes in salinity associated with the monsoon. During the summer months (May-October), strong southwesterly winds transport large quantities of water vapor to the Indian subcontinent. Much of the water precipitated over land is carried to the Bay of Bengal by the river systems. In the winter months (November-April), dry winds blow from the northeast. The volume of water discharged into the Bay of Bengal from the Ganges-Brahmaputra and Irrawaddy river systems during the summer is five times greater than during the winter (Rodolfo, 1967). The variation in fresh water input to the Bay of Bengal produces a seasonal salinity gradient in the surface waters. During the summer monsoon, a steep salinity gradient, increasing to the south, exists along $90^{\circ} \mathrm{E}$ (Wyrtki, 1973). This gradient forms primarily in response to riverine input, but is enhanced by the Southwest Monsoon Current which carries high salinity water into the central Bay of Bengal from the equatorial Indian Ocean (Prell et al., 1980). Sea-surface temperature (SST) variations in the Bay of Bengal are nearly constant over the year when compared to the fluctuations observed in the Arabian Sea (Wyrtki, 1973). During the winter monsoon, the average temperatures in the Bay of Bengal range from $26^{\circ} \mathrm{C}$ in the north, to $28^{\circ} \mathrm{C}$ in the south. During the summer monsoon, the temperatures are slightly higher and nearly isothermal, ranging from $28^{\circ} \mathrm{C}$ to $29^{\circ} \mathrm{C}$ (Wyrtki, 1973). In the southernmost reaches of the Bay of Bengal, the SST and the salinity show little variation throughout the year. In this region, near Site 758 , the SST is $28.5 \pm 1.0^{\circ} \mathrm{C}$ and the salinity is $33.8 \pm 0.3 \%$ throughout the year (Wyrtki, 1988). These values are typical of open ocean tropical regions.

Little is known about monsoon induced variations in biological productivity in the Bay of Bengal. Only in isolated locations within the Andaman Sea have distinct biological responses to monsoonal induced upwelling been documented (Colborn, 1975). During the late Quaternary, variations in biological productivity in the Andaman Sea have been related to the strength of the Northeast winter Monsoon (Fontugne and Duplessy, 1986). The variation of productivity recorded at Site 758 would largely depend on the Northeast Monsoon strength and associated equatorial current intensity.

\section{METHODS AND DATA}

\section{Stratigraphy}

Since sediment recovery gaps as large as $2.7 \mathrm{~m}$ occurred between almost every advanced piston core at Site 758, a composite depth section was constructed (Farrell and Janecek, this volume) by splicing together sedimentary intervals from Holes $758 \mathrm{~A}$ and $\mathrm{B}$ based on detailed correlations of high resolution stratigraphies such as magnetic susceptibility. The composite depth section provides a continuous record of the sedimentary sequence at Site 758. We used samples from Cores 121-758A-1H and $-2 \mathrm{H}$ which had recovery percentages of $102 \%$ and $104 \%$, respectively. A sedimentary interval of approximately $1.42 \mathrm{~m}$ representing approximately $60 \mathrm{k} . \mathrm{y}$. is missing between the two cores (see Fig. 4 in Farrell and Janecek, this volume). This gap, which contains oxygen isotope stage 10 (using the nomenclature of Imbrie et al., 1984), is successfully patched with the equivalent sedimentary interval from Core $121-758 \mathrm{~B}-1 \mathrm{H}$.

The chronostratigraphy used in this study is from Farrell and Janecek (this volume) and is primarily based on the correlation of the Site 758 oxygen isotope record from the planktonic foraminifer Globigerinoides sacculifer (300-355 $\mu \mathrm{m}$ size fraction) to the global average oxygen isotope record, the Spectral Mapping
(SPECMAP) stack (Imbrie et al., 1984; Prell et al., 1986). Age control is also provided by the position of the Brunhes/Matuyama paleomagnetic chron boundary (at 10.75 meters below sea floor (mbsf) which corresponds to $12.17 \mathrm{~m}$ on the composite depth scale). Our chronostratigraphy is consistent with the age constraints provided by the biostratigraphic datums established onboard (Shipboard Scientific Party, 1989) and with the disappearance of the pink variety of Globigerinoides ruber at 120 ka (Thompson et al., 1979) which occurs between Samples 121$758 \mathrm{~A}-1 \mathrm{H}-2,131 \mathrm{~cm}$ and $121 \mathrm{~cm}$.

\section{Faunal Analysis}

The 134 faunal samples used in this study are aliquots from the same samples used to determine $\% \mathrm{CaCO}_{3}$, coarse fraction, and stable isotope composition (Farrell and Janecek, this volume). The samples have an average spacing of $10 \mathrm{~cm}$, which is equivalent in time to $6 \mathrm{k} . \mathrm{y}$. The samples span from the core top $(0.01 \mathrm{~m})$ down to $13.83 \mathrm{~m}$ which corresponds to an age interval of $0-800$ ka. Preparation of the samples from the initial stages up to and including the separation of the $>150 \mu \mathrm{m}$ size fraction is described in Farrell and Janecek (this volume). Subsequent sample preparation and analysis follows the method outlined in Imbrie and Kipp (1971). Each sample is a split of the $>150 \mu \mathrm{m}$ fraction. Of the 134 samples, 124 contain at least 300 whole foraminifers. The remaining 10 samples have experienced greater dissolution and thus contain between 200 and 300 whole foraminifers. The relative abundances of 27 species of planktonic foraminifers were calculated for each sample and are listed in Table 1. The taxonomy of planktonic foraminifers used in this study is based on Parker (1962), Bé (1967), and Kipp (1976). This taxonomy was also used in previous studies of late Quaternary Indian Ocean foraminifers (e.g., Cullen, 1981; Cullen and Prell, 1984).

\section{RESULTS}

\section{Relative Abundance of Planktonic Foraminifers}

Descriptive statistics (Table 2) show that over the past 800 k.y., the foraminifer assemblages are dominated by six species which constitute $76 \%$ of the total planktonic foraminifer composition. In order of decreasing mean abundance, the six species are: $G$. ruber, Pulleniatina obliquiloculata, Neogloboquadrina dutertrei, Globorotalia menardii, G. sacculifer, and Globigerinita glutinata. These six species show larger downcore variations than the other species, as indicated by the larger standard deviations of the six.

Variations in the abundance of these six dominant species in the tropical Indian Ocean have previously been attributed to $\mathrm{CaCO}_{3}$ preservation and ecological factors (Cullen and Prell, 1984). G. ruber and G. sacculifer are major components of surface sediments underlying the warm waters of tropical oceans and are highly-susceptible to $\mathrm{CaCO}_{3}$ dissolution. G. glutinata is moderately-susceptible to dissolution, and is a dominant component in assemblages from regions influenced by upwelling, such as the western Arabian Sea and the northeastern Bay of Bengal (Cullen and Prell, 1984). The ecological role of this species is not well understood but it may be an ecotone between upwelling and tropical faunal assemblages (Hutson and Prell, 1980), or it may be associated with the margins of upwelling zones (Prell et al., 1990). P. obliquiloculata, N. dutertrei, and G. menardii are tropical species generally considered to be more resistant to dissolution (Berger, 1968; 1979; Parker and Berger, 1971; Adelseck, 1978; Thunell and Honjo, 1981a; b).N. dutertrei and G. menardii are highly abundant in regions characterized by a relatively shallow thermocline, intense upwelling, and high productivity (Fairbanks et al., 1982; Thunell and Reynolds, 1984). These species are especially abundant in strong current systems near continental 
margins (Bé and Tolderlund, 1971). The ecological niche of $P$ obliquiloculata is unclear. The high abundance of this species in the western-central tropical Pacific suggests that it prefers relatively warmer SST and/or a depressed thermocline. Nevertheless, in samples from the tropical Atlantic Ocean (Ravelo et al., 1990), this species occurred with $N$. dutertrei and $G$. menardii which prefer a shallow thermocline.

Globigerina bulloides and Globorotalia tumida are relatively minor but important components of the foraminifer assemblages because respectively, they are strongly linked to ecological and preservational conditions. G. bulloides is an important component of subpolar-transitional faunas (Parker and Berger, 1971; Kipp, 1976; Hutson and Prell, 1980) and is abundant in low latitude upwelling regions such as the Arabian Sea (Hutson and Prell, 1980; Prell and Curry, 1981; Prell, 1984; Cullen and Prell, 1984). $G$. tumida is most abundant in samples from the deepest waters and is quite rare in shallow water sites (Cullen and Prell, 1984). This observation is consistent with the conclusion that $G$. tumida is one of the most dissolution-resistant foraminifer species (Berger, 1968; 1979; Parker and Berger, 1971; Adelseck, 1978; Thunell and Honjo, 1981a; b).

\section{Temporal Variation of Planktonic Foraminifers}

The relative percentages of the species in the core top sample $(121-758 \mathrm{~A}-1 \mathrm{H}-1,1 \mathrm{~cm})$ from Site 758 are quite similar to percentages observed in nearby surface sediment samples (Cullen and Prell, 1984). Downcore faunal assemblages at Site 758 can be interpreted with respect to assemblages from modern sediments (Cullen and Prell, 1984; Prell, 1985) because of the similarity in core top results. Fluctuations in relative abundance of eight major foraminifer species are presented with respect to age (Fig. 2). The eight species include $G$. bulloides and G. tumida, in addition to the six dominant species previously discussed. Particularly noteworthy among the trends shown in Figure 2 are:

1. The pulse of decreased abundances of G. ruber, G. sacculifer, G. bulloides, and G. glutinata between 490 and $550 \mathrm{ka}$, and the concomitant increase in the abundance of $P$. obliquiloculata, G. menardii, and G. tumida.

2. A long-term cycle in $G$. tumida abundance characterized by low values from 700 to $800 \mathrm{ka}$ and from 0 to $10 \mathrm{ka}$, and high values centered between 400 and $550 \mathrm{ka}$.

3. Intervals, such as between 490 and $550 \mathrm{ka}$, in which the abundance of $P$. obliquiloculata was much greater than the maximum value of $21 \%$ observed in surface sediments of the North Indian Ocean (Cullen and Prell, 1984).

\section{Temporal Variations in the Resistant Species Ratio (RSP\%)}

Variations in foraminifer assemblages reflect changes in preservation on the sea floor, as well as ecological changes at the sea surface. The preservation of planktonic foraminifers in the deep sea is controlled by the saturation state of the bottom waters and the interstitial pore waters with respect to calcium carbonate, which in turn is a function of overall mixing rate and fertility (Berger, 1979). Quantitative (Thunell and Honjo, 1981a; b) and semi-quantitative (Adelseck, 1978; Berger, 1968; 1979; Parker and Berger, 1971; Malmgren, 1983; Peterson and Prell, 1985a) field and laboratory studies have established a relative ranking of the degree to which planktonic foraminifer species are susceptible to $\mathrm{CaCO}_{3}$ dissolution. For example, in surface sediments from the eastern equatorial Indian Ocean, Peterson and Prell (1985a) observed a systematic increase in the abundance of foraminifer species with thick skeletons, such as G. menardii, G. tumida, and $N$. dutertrei, with increasing water depth. The abundance of the dissolution resistant species increases at the expense of less resistant species. In deep water samples, the assemblage contains a reduced number of specimens from the species which are moderately-susceptible to dissolution, such as G. glutinata, $N$. hexagona, G. bulloides, and G. calida, and even fewer specimens of the highly-susceptible species such as G. ruber and G. sacculifer.

The foraminifer lysocline (FL) is the water depth which separates well-preserved from poorly-preserved foraminifer assemblages (Berger, 1975). The depth of the FL in the Indian Ocean has been determined quantitatively based on noticeable changes in the resistant species ratio (RSP) (Cullen and Prell, 1984). The RSP\% is defined (Cullen and Prell, 1984) as the sum of the percentages of the following 14 dissolution-resistant species and varieties: G. menardii, N. dutertrei, P.obliquiloculata, G. tumida, $S$. dehiscens, "pachyderma-dutertrei" intergrade, G. crassaformis, G. menardii neoflexuosa, $G$. pachyderma right coiling, $G$. truncatulinoides right coiling, $G$. pachyderma left coiling, $G$. inflata, G. truncatulinoides left coiling, and G. humilis. In our calculation of the RSP\%, we grouped G. menardii neoflexuosa with $G$. menardii, and we excluded $G$. pachyderma left coiling and $G$. humilis because they are absent from the assemblages. The FL has been equated with the water depth where the RSP level is equal to $30 \%$ (Cullen and Prell, 1984). Values greater than $30 \%$ are considered to lie beneath the FL. Consistent with the original definition of RSP\%, the variations in faunal assemblages with RSP\% greater than $30 \%$ are considered to be significantly influenced by $\mathrm{CaCO}_{3}$ dissolution. Assemblages with RSP\% values less than $30 \%$ lie above the FL and variations in these assemblages are thought to primarily reflect fluctuations in foraminifer input from the surface waters, and thus ecological factors. Nevertheless, some dissolution does occur above the FL (Peterson and Prell, 1985a). In the Bay of Bengal, the FL deepens from $\sim 2200 \mathrm{~m}$ in the northernmost regions, to $2750 \mathrm{~m}$ in the south (Cullen and Prell, 1984). According to this estimate, the depth of Site 758 (2924 m) is presently located slightly below the FL. This estimate is consistent with the Site 758 core top RSP value of $36 \%$.

In Figure 3, the RSP\% of the samples from Site 758 is compared to the coarse fraction and to $\delta^{18} \mathrm{O}$. Coarse fraction is defined as the wt $\%$ of a sample $>150 \mathrm{~mm}$. Coarse fraction is a reliable index of relative dissolution intensity in many regions (Berger, 1970; Berger et al., 1982). Furthermore, since coarse fraction is a lithologic index rather than a faunal index, it is an independent proxy indicator of dissolution. Low coarse fraction values are interpreted as times of enhanced dissolution. The oxygen isotope record is a proxy indicator of global ice volume (Shackleton and Opdyke, 1973; 1976) and is thus used to identify glacial and interglacial climate stages (Imbrie et al., 1984; Prell et al., 1986). As expected, a negative correlation exists between RSP\% and coarse fraction (Fig. 4) since dissolution is thought to control both parameters. The correlation is noisy for several reasons. First, RSP\% is controlled by ecological factors as well as by dissolution. Second, RSP\% is probably more sensitive than coarse fraction to subtle changes in the corrosiveness of the water. If this is the case, the dissolution response of RSP\% would lead the coarse fraction response. In addition, the magnitude of the RSP\% response is not necessarily equal to the magnitude of the coarse fraction response. The increase of RSP\% in the downcore sample is mainly attributed to the increase of differential solution of foraminifer assemblages. The most severe dissolution is indicated by the highest RSP\% and the lowest coarse fractions. Likewise, the best preservation is indicated by the lowest RSP\% and the highest coarse fractions. Using the narrow definition of the FL as the $30 \%$ RSP level, the downcore RSP\% results indicate that Site 758 has remained beneath the FL during most of the past $800 \mathrm{ka}$. The only times during which the FL deepened to or below the depth of Site $758(2924 \mathrm{~m})$ was during the latter half of glacial stages and during transitions from glacials to interglacials. For example, the 
Table 1. Planktonic foraminifer percentage data in the composite depth section from Hole 758A. Foraminiferal codes are listed in Table 2.

\begin{tabular}{|c|c|c|c|c|c|c|}
\hline $\begin{array}{l}\text { Core, section } \\
\text { interval }(\mathrm{cm})\end{array}$ & $\begin{array}{l}\text { Composite } \\
\text { depth }(m)\end{array}$ & $\begin{array}{l}\text { Age } \\
(\mathrm{Ma})\end{array}$ & O.unive & G.cglob & G.ruber & G.tenel \\
\hline $1 \mathrm{H}-1,1$ & 0.01 & 0.001 & 0.895 & 4.251 & 21.924 & 0.000 \\
\hline $1 \mathrm{H}-1,11$ & 0.11 & 0.006 & 0.619 & 2.474 & 32.165 & 0.412 \\
\hline $1 \mathrm{H}-1,21$ & 0.21 & 0.010 & 0.781 & 0.312 & 35.156 & 0.156 \\
\hline $1 \mathrm{H}-1,31$ & 0.31 & 0.015 & 0.138 & 0.688 & 35.488 & 1.651 \\
\hline $1 \mathrm{H}-1,41$ & 0.41 & 0.019 & 0.840 & 2.353 & 21.344 & 1.849 \\
\hline $1 \mathrm{H}-1,51$ & 0.51 & 0.024 & 0.325 & 2.602 & 12.520 & 0.325 \\
\hline $1 \mathrm{H}-1,61$ & 0.61 & 0.029 & 0.723 & 1.627 & 17.722 & 0.000 \\
\hline $1 \mathrm{H}-1,71$ & 0.71 & 0.034 & 0.369 & 2.214 & 15.498 & 0.000 \\
\hline $1 \mathrm{H}-1,81$ & 0.81 & 0.038 & 0.731 & 1.316 & 17.251 & 1.316 \\
\hline IH-1, 91 & 0.91 & 0.043 & 0.883 & 2.297 & 12.544 & 0.177 \\
\hline $1 \mathrm{H}-1,101$ & 1.01 & 0.048 & 0.303 & 1.061 & 15.909 & 0.758 \\
\hline $1 \mathrm{H}-1,111$ & 1.11 & 0.053 & 0.955 & 1.274 & 13.057 & 1.274 \\
\hline $1 \mathrm{H}-\mathrm{I}, 12 \mathrm{I}$ & 1.21 & 0.058 & 0.476 & 0.714 & 18.809 & 0.952 \\
\hline $1 \mathrm{H}-1,131$ & 1.31 & 0.063 & 0.829 & 1.303 & 22.512 & 0.356 \\
\hline $1 \mathrm{H}-\mathrm{I}, 141$ & 1.41 & 0.067 & 0.439 & 2.339 & 14.328 & 2.339 \\
\hline $1 \mathrm{H}-2,1$ & 1.51 & 0.071 & 0.484 & 0.000 & 10.896 & 3.632 \\
\hline $1 \mathrm{H}-2,11$ & 1.61 & Ash A & & & & \\
\hline $1 \mathrm{H}-2,2 \mathrm{l}$ & 1.71 & Ash A & & & & \\
\hline $1 \mathrm{H}-2,31$ & 1.81 & Ash A & & 1.375 & & 1.031 \\
\hline $\begin{array}{l}1 \mathrm{H}-2,41 \\
1 \mathrm{H}-2,51\end{array}$ & $\begin{array}{l}1.91 \\
2.01\end{array}$ & $\begin{array}{l}0.076 \\
\text { Ash B }\end{array}$ & 0.344 & & 9.900 & 1.031 \\
\hline $1 \mathrm{H}-2,61$ & 2.11 & 0.079 & 0.225 & 0.899 & 15.506 & 0.449 \\
\hline $1 \mathrm{H}-2,71$ & 2.21 & 0.080 & 1.571 & 1.745 & 24.084 & 0.349 \\
\hline $1 \mathrm{H}-2,81$ & 2.31 & 0.089 & 0.536 & 3.393 & 12.321 & 0.893 \\
\hline $1 \mathrm{H}-2,91$ & 2.41 & 0.099 & 0.000 & 2.321 & 13.036 & 0.893 \\
\hline $1 \mathrm{H}-2,101$ & 2.51 & 0.107 & 0.536 & 2.681 & 11.528 & 0.000 \\
\hline $1 \mathrm{H}-2,111$ & 2.61 & 0.114 & 0.509 & 1.019 & 10.526 & 0.340 \\
\hline $1 \mathrm{H}-2,121$ & 2.71 & 0.122 & 0.000 & 2.832 & 7.407 & 0.871 \\
\hline $1 \mathrm{H}-2,131$ & 2.81 & 0.126 & 0.338 & 1.239 & 28.604 & 0.338 \\
\hline $1 \mathrm{H}-2,141$ & 2.91 & 0.131 & 0.976 & 1.301 & 21.626 & 1.626 \\
\hline $1 \mathrm{H}-3,1$ & 3.01 & 0.135 & 0.529 & 0.741 & 14.180 & 2.434 \\
\hline IH-3, 11 & 3.11 & 0.142 & 1.024 & 1.536 & 23.550 & 0.341 \\
\hline $1 \mathrm{H}-3,2 \mathrm{I}$ & 3.21 & 0.149 & 0.879 & 0.977 & 29.199 & 0.977 \\
\hline $1 \mathrm{H}-3,31$ & 3.31 & 0.157 & 1.844 & 1.928 & 26.152 & 1.676 \\
\hline $1 \mathrm{H}-3,41$ & 3.41 & 0.164 & 0.446 & 2.232 & 16.518 & 0.893 \\
\hline $1 \mathrm{H}-3,51$ & 3.51 & 0.171 & 0.629 & 1.048 & 18.868 & 0.839 \\
\hline IH-3, 61 & 3.61 & 0.177 & 0.890 & 2.671 & 14.243 & 0.594 \\
\hline $1 \mathrm{H}-3,71$ & 3.71 & 0.183 & 0.382 & 1.721 & 16.826 & 0.765 \\
\hline $1 \mathrm{H}-3,81$ & 3.81 & 0.189 & 0.209 & 2.511 & 10.670 & 0.837 \\
\hline $1 \mathrm{H}-3,91$ & 3.91 & 0.194 & 0.704 & 1.408 & 16.667 & 0.235 \\
\hline $1 \mathrm{H}-3,101$ & 4.01 & 0.205 & 0.325 & 1.948 & 5.520 & 0.000 \\
\hline $1 \mathrm{H}-3,111$ & 4.11 & 0.211 & 0.000 & 0.848 & 13.136 & 0.636 \\
\hline $1 \mathrm{H}-3,121$ & 4.21 & 0.218 & 0.000 & 2.169 & 16.145 & 0.482 \\
\hline $1 \mathrm{H}-3,131$ & 4.31 & 0.223 & 0.592 & 2.071 & 18.935 & 0.296 \\
\hline $1 \mathrm{H}-3,141$ & 4.41 & 0.228 & 0.338 & 2.196 & 16.892 & 0.338 \\
\hline IH-4, I & 4.51 & 0.232 & 0.480 & 0.480 & 10.791 & 0.959 \\
\hline $1 \mathrm{H}-4,11$ & 4.61 & 0.237 & 0.516 & 1.289 & 18.557 & 0.000 \\
\hline $\mathrm{IH}-4,21$ & 4.71 & 0.240 & 0.957 & 0.239 & 32.297 & 0.239 \\
\hline IH $-4,31$ & 4.81 & 0.244 & 0.442 & 0.736 & 19.588 & 0.147 \\
\hline $1 \mathrm{H}-4,41$ & 4.91 & 0.247 & 0.857 & 0.857 & 13.714 & 0.857 \\
\hline $1 \mathrm{H}-4,51$ & 5.01 & 0.252 & 0.000 & 0.993 & 12.252 & 0.993 \\
\hline $1 \mathrm{H}-4,61$ & 5.11 & 0.257 & 0.699 & 0.466 & 17.016 & 0.466 \\
\hline $1 \mathrm{H}-4,71$ & 5.21 & 0.263 & 0.194 & 0.583 & 20.777 & 0.777 \\
\hline $1 \mathrm{H}-4,81$ & 5.31 & 0.268 & 0.398 & 2.783 & 24.056 & 0.795 \\
\hline $1 \mathrm{H}-3,81$ & 5.32 & 0.269 & 1.587 & 1.587 & 27.381 & 0.397 \\
\hline $1 \mathrm{H}-3,91$ & 5.42 & 0.278 & 0.000 & 0.000 & 23.673 & 0.664 \\
\hline $1 \mathrm{H}-3,101$ & 5.52 & 0.287 & 0.000 & 0.356 & 15.303 & 1.779 \\
\hline $1 \mathrm{H}-3,111$ & 5.62 & 0.295 & 0.000 & 0.329 & 18.092 & 0.329 \\
\hline $\mid \mathrm{H}-3,12 \mathrm{I}$ & 5.72 & 0.301 & 0.298 & 1.190 & 16.964 & 0.893 \\
\hline $1 \mathrm{H}-3,13 \mathrm{I}$ & 5.82 & 0.306 & 1.094 & 0.000 & 17.068 & 2.188 \\
\hline $1 \mathrm{H}-3,141$ & 5.92 & 0.311 & 0.932 & 0.699 & 12.121 & 0.466 \\
\hline $1 \mathrm{H}-4,1$ & 6.02 & 0.316 & 1.556 & 1.167 & 11.673 & 1.167 \\
\hline $1 \mathrm{H}-4,1]$ & 6.12 & 0.321 & 2.333 & 1.333 & 12.333 & 0.000 \\
\hline $1 \mathrm{H}-4,21$ & 6.22 & 0.326 & 1.678 & 1.007 & 10.067 & 0.336 \\
\hline $1 \mathrm{H}-4,31$ & 6.32 & 0.331 & 1.181 & 0.394 & 17.323 & 0.000 \\
\hline $1 \mathrm{H}-4,41$ & 6.42 & 0.334 & 0.959 & 0.719 & 33.573 & 0.240 \\
\hline $1 \mathrm{H}-4,51$ & 6.52 & 0.338 & 1.399 & 0.233 & 35.198 & 1.166 \\
\hline $1 \mathrm{H}-4,61$ & 6.62 & 0.341 & 2.344 & 0.391 & 18.359 & 3.125 \\
\hline $1 \mathrm{H}-4,71$ & 6.72 & 0.349 & 0.926 & 0.463 & 15.278 & 3.241 \\
\hline $1 \mathrm{H}-4,81$ & 6.82 & 0.357 & 1.412 & 0.706 & 21.882 & 0.471 \\
\hline $1 \mathrm{H}-4,91$ & 6.92 & 0.365 & 1.681 & 0.840 & 18.067 & 0.000 \\
\hline $1 \mathrm{H}-4,101$ & 7.02 & 0.373 & 0.692 & 1.038 & 20.069 & 0.000 \\
\hline $1 \mathrm{H}-4,111$ & 7.12 & 0.381 & 1.152 & 1.152 & 22.811 & 0.691 \\
\hline $1 \mathrm{H}-4,12 \mathrm{I}$ & 7.22 & 0.388 & 1.026 & 0.513 & 23.846 & 0.256 \\
\hline $1 \mathrm{H}-4,131$ & 7.32 & 0.396 & 0.418 & 0.418 & 6.695 & 0.000 \\
\hline $1 \mathrm{H}-4,141$ & 7.42 & 0.404 & 0.000 & 0.518 & 12.435 & 2.591 \\
\hline $2 \mathrm{H}-1,1$ & 7.43 & 0.405 & 1.506 & 0.301 & 17.470 & 0.000 \\
\hline $2 \mathrm{H}-1,11$ & 7.53 & 0.419 & 0.781 & 2.734 & 10.928 & 0.000 \\
\hline $2 \mathrm{H}-1,2 \mathrm{I}$ & 7.63 & 0.434 & 1.282 & 2.564 & 21.154 & 2.137 \\
\hline $2 \mathrm{H}-1,31$ & 7.73 & 0.443 & 1.724 & 2.874 & 19.157 & 1.149 \\
\hline $2 \mathrm{H}-1,4 \mathrm{I}$ & 7.83 & 0.452 & 1.695 & 0.726 & 19.855 & 1.211 \\
\hline $2 \mathrm{H}-1,5 \mathrm{I}$ & 7.93 & 0.460 & 1.029 & 1.323 & 21.618 & 1.471 \\
\hline $2 \mathrm{H}-1,61$ & 8.03 & 0.469 & 0.612 & 1.835 & 18.349 & 0.306 \\
\hline $2 \mathrm{H}-1,71$ & 8.13 & 0.478 & 1.025 & 2.459 & 14.754 & 1.025 \\
\hline $2 \mathrm{H}-1,81$ & 8.23 & 0.491 & 0.533 & 0.000 & 16.800 & 2.400 \\
\hline $2 \mathrm{H}-1,9 \mathrm{I}$ & 8.33 & 0.513 & 1.345 & 1.345 & 3.139 & 0.000 \\
\hline $2 \mathrm{H}-1,101$ & 8.43 & 0.525 & 0.000 & 0.000 & 7.407 & 0.463 \\
\hline $2 \mathrm{H}-1,111$ & 8.53 & Ash C & & & & \\
\hline $2 \mathrm{H}-1,121$ & 8.63 & 0.538 & 2.051 & 0.000 & 6.667 & 0.000 \\
\hline $2 \mathrm{H}-1,131$ & 8.73 & 0.552 & 0.943 & 0.943 & 12.264 & 4.717 \\
\hline $2 \mathrm{H}-1,141$ & 8.83 & 0.556 & 1.303 & 2.606 & 16.287 & 0.000 \\
\hline $2 \mathrm{H}-2,1$ & 8.93 & 0.559 & 1.194 & 0.000 & 20.597 & 0.896 \\
\hline
\end{tabular}


Table 1 (continued).

\begin{tabular}{|c|c|c|c|c|c|}
\hline $\begin{array}{l}\text { Core, section } \\
\text { Interval (cm) }\end{array}$ & $\begin{array}{c}\text { Composite } \\
\text { depth(m) }\end{array}$ & $\begin{array}{l}\text { Age } \\
(\mathrm{Ma})\end{array}$ & G.cglom & G.hexag & P.obliq \\
\hline $1 \mathrm{H}-1,1$ & 0.01 & 0.001 & 4.698 & 1.119 & 9.620 \\
\hline $1 \mathrm{H}-1,11$ & 0.11 & 0.006 & 3.711 & 2.474 & 7.835 \\
\hline $1 \mathrm{H}-1,21$ & 0.21 & 0.010 & 1.875 & 2.031 & 7.188 \\
\hline $1 \mathrm{H}-1,131$ & 0.31 & 0.015 & 0.688 & 3.439 & 8.253 \\
\hline $1 \mathrm{H}-1,41$ & 0.41 & 0.019 & 2.857 & 1.681 & 12.437 \\
\hline $1 \mathrm{H}-1,51$ & 0.51 & 0.024 & 2.114 & $1.9 \mathrm{~S} 1$ & 13.333 \\
\hline $1 \mathrm{H}-1,61$ & 0.61 & 0.029 & 3.255 & 1.989 & 11.754 \\
\hline $1 \mathrm{H}-1,71$ & 0.71 & 0.034 & 6.273 & 1.107 & 18.819 \\
\hline $1 \mathrm{H}-1,91$ & 0.91 & 0.043 & 3.003 & 1.590 & 26.855 \\
\hline $1 \mathrm{H}-1,101$ & 1.01 & 0.048 & 3.030 & 2.121 & 18.030 \\
\hline $\mathrm{H}-1,111$ & 1.11 & 0.053 & 4.140 & 1.274 & 21.338 \\
\hline $1 \mathrm{H}-1,121$ & 1.21 & 0.058 & 4.524 & 0.476 & 20.476 \\
\hline $1 \mathrm{H}-1,131$ & 1.31 & 0.063 & 5.569 & 2.014 & 14.100 \\
\hline $\mathrm{H}-\mathrm{I}, 14 \mathrm{I}$ & 1.41 & 0.067 & 4.971 & 1.608 & 14.181 \\
\hline $1 \mathrm{H}-2,1$ & 1,51 & 0.071 & 3.632 & 0.242 & 11.864 \\
\hline $1 \mathrm{H}-2,11$ & 1.61 & Ash A & & & \\
\hline $1 \mathrm{H}-2,21$ & 1.71 & Ash A & & & \\
\hline $1 \mathrm{H}-2,31$ & 1.81 & AshA & & & \\
\hline IH $-2,41$ & 1.91 & 0.076 & 8.591 & 1.031 & 18.557 \\
\hline $1 \mathrm{H}-2,61$ & 2.11 & 0.079 & 5.843 & 1.124 & 13.933 \\
\hline $1 \mathrm{H}-2,71$ & 2.21 & 0.080 & 6.108 & 1.047 & 12.565 \\
\hline $1 \mathrm{H}-2,81$ & 2.31 & 0.089 & 7.321 & 0.714 & 13.036 \\
\hline $1 \mathrm{H}-2,91$ & 2.41 & 0.099 & 5.714 & 1.429 & 9.464 \\
\hline $1 \mathrm{H}-2,101$ & 2.51 & 0.107 & 6.702 & 0.536 & 10.992 \\
\hline $1 \mathrm{H}-2,111$ & 2.61 & 0.114 & 2.886 & 0.849 & 14.601 \\
\hline $1 \mathrm{H}-2,121$ & 2.71 & 0.122 & 4.357 & 1.089 & 10.240 \\
\hline $1 \mathrm{H}-2,131$ & 2.81 & 0.126 & 2.590 & 3.378 & 5.180 \\
\hline $1 \mathrm{H}-2,14 \mathrm{I}$ & 2.91 & 0.131 & 4.390 & 1.301 & 9.919 \\
\hline $1 \mathrm{H}-3, \mathrm{I}$ & 3.01 & 0.135 & 2.646 & 1.270 & 12.698 \\
\hline IH-3, II & 3.11 & 0.142 & 4.607 & 2.218 & 10.580 \\
\hline $1 \mathrm{H}-3,21$ & 3.21 & 0.149 & 4.883 & 2.832 & 9.473 \\
\hline $1 \mathrm{H}-3,31$ & 3.31 & 0.157 & 3.772 & 1.760 & 7.963 \\
\hline $1 \mathrm{H}-3,41$ & 3.41 & 0.164 & 3.571 & 1.786 & 11.384 \\
\hline $1 \mathrm{H}-3,51$ & 3.51 & 0.171 & 2.306 & 3.145 & 10.482 \\
\hline $1 \mathrm{H}-3,61$ & 3.61 & 0.177 & 2.374 & 2.374 & 12.463 \\
\hline $1 \mathrm{H}-3,71$ & 3.71 & 0.183 & 2.868 & 0.574 & 8.031 \\
\hline $1 \mathrm{H}-3,81$ & 3.81 & 0.189 & 2.720 & 1.046 & 18.619 \\
\hline $1 \mathrm{H}-3,91$ & 3.91 & 0.194 & 9.390 & 1.174 & 17.606 \\
\hline $1 \mathrm{H}-3,101$ & 4.01 & 0.205 & 7.143 & 0.325 & 17.208 \\
\hline $1 \mathrm{H}-3,111$ & 4.11 & 0.211 & 4.873 & 0.636 & 13.983 \\
\hline $1 \mathrm{H}-3,121$ & 4.21 & 0.218 & 2.892 & 1.928 & 9.398 \\
\hline $1 \mathrm{H}-3,131$ & 4.31 & 0.223 & 5.325 & 0.888 & 10.059 \\
\hline $1 \mathrm{H}-3,141$ & 4.41 & 0.228 & 6.081 & 2.027 & 11.318 \\
\hline $1 \mathrm{H}-4,1$ & 4.51 & 0.232 & 2.398 & 0.959 & 14.628 \\
\hline $1 \mathrm{H}-4, \mathrm{II}$ & 4.61 & 0.237 & 6.701 & 0.773 & 10.567 \\
\hline$|\mathrm{H}-4,2|$ & 4.71 & 0.240 & 2.632 & 2.153 & 11.244 \\
\hline $1 \mathrm{H}-4,31$ & 4.81 & 0.244 & 3.240 & 1.325 & 11.782 \\
\hline $1 \mathrm{H}-4,41$ & 4.91 & 0.247 & 1.571 & 1.143 & 16.714 \\
\hline $1 \mathrm{H}-4,51$ & 5.01 & 0.252 & 2.318 & 1.324 & 12.914 \\
\hline $1 \mathrm{H}-4,61$ & 5.11 & 0.257 & 2.564 & 0.233 & 13.054 \\
\hline $1 \mathrm{H}-4,71$ & 5.21 & 0.263 & 3.883 & 1.359 & 8.544 \\
\hline $1 \mathrm{H}-4,81$ & 5.31 & 0.268 & 6.561 & 1.392 & 11.133 \\
\hline $1 \mathrm{H}-3,81$ & 5.32 & 0.269 & 5.556 & 2.381 & 9.127 \\
\hline $1 \mathrm{H}-3,91$ & 5.42 & 0.278 & 1.549 & 0.664 & 9.292 \\
\hline $1 \mathrm{H}-3,101$ & 5.52 & 0.287 & 2.847 & 0.356 & 9.609 \\
\hline $1 \mathrm{H}-3,111$ & 5.62 & 0.295 & 4.276 & 1.645 & 7.566 \\
\hline $1 \mathrm{H}-3,121$ & 5.72 & 0.301 & 6.250 & 2.083 & 15.179 \\
\hline $1 \mathrm{H}-3,13 \mathrm{I}$ & 5.82 & 0.306 & 2.845 & 0.656 & 9.847 \\
\hline $\mid \mathrm{H}-3,141$ & 5.92 & 0.311 & 2.564 & 0.466 & 11.422 \\
\hline $1 \mathrm{H}-4,1$ & 6.02 & 0.316 & 3.891 & 0.778 & 14.397 \\
\hline $1 \mathrm{H}-4,1 \mathrm{I}$ & 6.12 & 0.321 & 9.333 & 1.000 & 16.333 \\
\hline $1 \mathrm{H}-4,21$ & 6.22 & 0.326 & 3.020 & 0.000 & 20.470 \\
\hline $1 \mathrm{H} 4,31$ & 6.32 & 0.331 & 5.905 & 2.362 & 11.024 \\
\hline $1 \mathrm{H} 4,41$ & 6.42 & 0.334 & 4.077 & 4.316 & 5.516 \\
\hline $1 \mathrm{H} 4,51$ & 6.52 & 0.338 & 3.730 & 1.865 & 5.361 \\
\hline $1 \mathrm{H} 4,61$ & 6.62 & 0.341 & 1.172 & 0.000 & 9.375 \\
\hline $1 \mathrm{H} 4,7 \mathrm{I}$ & 6.72 & 0.349 & 1.852 & 0.926 & 10.185 \\
\hline $1 \mathrm{H} 4,81$ & 6.82 & 0.357 & 1.412 & 3.059 & 12.235 \\
\hline IH 4,91 & 6.92 & 0.365 & 8.824 & 1.681 & 14.706 \\
\hline $1 \mathrm{H}-4,101$ & 7.02 & 0.373 & 4.844 & 0.692 & 19.723 \\
\hline $1 \mathrm{H} 4,111$ & 7.12 & 0.381 & 3.456 & 1.152 & 18.664 \\
\hline $1 \mathrm{H} 4,121$ & 7.22 & 0.388 & 3.846 & 0.000 & 14.615 \\
\hline $1 \mathrm{H} 4,131$ & 7.32 & 0.396 & 0.837 & 0.418 & 18.410 \\
\hline $1 \mathrm{H} 4,141$ & 7.42 & 0.404 & 1.554 & 1.036 & 15.026 \\
\hline $2 \mathrm{H}-1,1$ & 7.43 & 0.405 & 4.518 & 1.506 & 15.964 \\
\hline $2 \mathrm{H}-\mathrm{I}, \mathrm{II}$ & 7.53 & 0.419 & $6.64 t$ & 0.391 & 13.281 \\
\hline $2 \mathrm{H}-1,2 \mathrm{I}$ & 7.63 & 0.434 & 3.205 & 1.068 & 8.333 \\
\hline $2 \mathrm{H}-1,3 \mathrm{l}$ & 7.73 & 0.443 & 4.406 & 1.341 & 12.835 \\
\hline $2 \mathrm{H}-1,41$ & 7.83 & 0.452 & 3.874 & 1.453 & 14.286 \\
\hline $2 \mathrm{H}-1,1$ & 7.93 & 0.460 & 2.794 & 1.323 & 9.853 \\
\hline $2 \mathrm{H}-1,6 \mathrm{I}$ & 8.03 & 0.469 & 5.199 & 0.917 & 14.373 \\
\hline $2 \mathrm{H}-1,71$ & 8.13 & 0.478 & 3.074 & 0.820 & 16.598 \\
\hline $2 \mathrm{H}-1,81$ & 8.23 & 0.491 & 2.667 & 0.533 & 20.533 \\
\hline $2 \mathrm{H}-1,91$ & 8.33 & 0.513 & 1.345 & 0.897 & 33.632 \\
\hline $2 \mathrm{H}-1,10 \mathrm{I}$ & 8.43 & 0.525 & 2,778 & 0.000 & 30.093 \\
\hline $2 \mathrm{H}-1,11 \mathrm{I}$ & 8.53 & Ash C & & & \\
\hline $2 \mathrm{H}-1,12 \mathrm{I}$ & 8.63 & 0.538 & 1.539 & 0.000 & 38.461 \\
\hline $2 \mathrm{H}-1,131$ & 8.73 & 0.552 & 2.201 & 0.629 & 21.384 \\
\hline $2 \mathrm{H}-1,141$ & 8.83 & 0.556 & 2.280 & 0.977 & 22.476 \\
\hline $2 \mathrm{H}-2,1$ & 8.93 & 0.559 & 2.388 & 2.687 & 22.388 \\
\hline
\end{tabular}


Table 1 (continued).

\begin{tabular}{|c|c|c|c|c|c|c|c|c|c|c|c|c|c|c|c|c|}
\hline $\begin{array}{l}\text { Core, section } \\
\text { interval }(\mathrm{cm})\end{array}$ & $\begin{array}{l}\text { Composite } \\
\text { depth (m) }\end{array}$ & $\begin{array}{l}\text { Age } \\
(\mathrm{Ma})\end{array}$ & O.unive & G.cglob & G.ruber & G.tenel & G.saccu & S.dehis & G.aequi & G.calid & G.bullo & G.falco & G.digit & G.rubes & G.pac R & N.duter \\
\hline $2 \mathrm{H}-2,11$ & 9.03 & 0.563 & 1.592 & 0.531 & 25.995 & 4.244 & 5.570 & 0.531 & 1.592 & 2.387 & 4.244 & 0.531 & 0.000 & 1.592 & 0.000 & 14.058 \\
\hline $2 \mathrm{H}-2,21$ & 9.13 & 0.566 & 0.552 & 0.735 & 17.647 & 3.125 & 7.169 & 0.184 & 2.206 & 1.471 & 4.412 & 0.368 & 0.184 & 0.368 & 0.184 & 21.324 \\
\hline $2 \mathrm{H}-2,31$ & 9.23 & 0.569 & 0.662 & 0.331 & 7.616 & 0.000 & 8.278 & 2.318 & 5.960 & 0.662 & 3.311 & 0.000 & 0.331 & 0.000 & 0.000 & 26.159 \\
\hline $2 \mathrm{H}-2,4 \mathrm{I}$ & 9.33 & 0.572 & 0.000 & 0.796 & 5.570 & 0.000 & 6.897 & 1.326 & 4.244 & 0.531 & 3.448 & 0.265 & 0.796 & 0.000 & 0.531 & 16.976 \\
\hline $2 \mathrm{H}-2,51$ & 9.43 & 0.576 & 0.926 & 2.315 & 4.630 & 0.000 & 11.111 & 1.620 & 4.398 & 3.241 & 9.491 & 0.694 & 1.157 & 0.000 & 0.000 & 14.120 \\
\hline $2 \mathrm{H}-2,6 \mathrm{I}$ & 9.53 & 0.581 & 1.527 & 2.290 & 9.733 & 0.000 & 8.206 & 0.763 & 3.244 & 1.908 & 11.832 & 0.763 & 1.336 & 0.000 & 0.000 & 17.939 \\
\hline $2 \mathrm{H}-2,7 \mathrm{I}$ & 9.63 & 0.585 & 0.510 & 0.510 & 11.395 & 1.361 & 5.442 & 0.510 & 1.701 & 1.020 & 7.993 & 0.510 & 0.170 & 0.000 & 0.000 & 15.986 \\
\hline $2 \mathrm{H}-2,81$ & 9.73 & 0.590 & 0.370 & 0.741 & 13.333 & 3.148 & 7.407 & 0.741 & 2.037 & 2.222 & 8.333 & 0.741 & 0.185 & 0.185 & 0.000 & 23.704 \\
\hline $2 \mathrm{H}-2,91$ & 9.83 & 0.596 & 0.397 & 0.397 & 10.714 & 0.397 & 7.540 & 1.587 & 1.984 & 1.190 & 9.921 & 0.000 & 0.794 & 0.000 & 0.000 & 14.286 \\
\hline $2 \mathrm{H}-2,101$ & 9.93 & 0.607 & 1.132 & 0.755 & 5.283 & 0.000 & 10.189 & 1.887 & 3.774 & 2.642 & 7.925 & 1.132 & 0.755 & 0.000 & 0.000 & 13.585 \\
\hline $2 \mathrm{H}-2,11 \mathrm{I}$ & 10.03 & 0.617 & 1.136 & 0.568 & 16.477 & 1.420 & 12.216 & 1.136 & 2.841 & 2.841 & 11.080 & 0.568 & 0.568 & 0.000 & 0.000 & 15.909 \\
\hline $2 \mathrm{H}-2,12 \mathrm{I}$ & 10.13 & 0.623 & 0.388 & 0.194 & 24.272 & 1.942 & 11.262 & 0.388 & 2.524 & 2.718 & 6.408 & 0.777 & 0.388 & 0.000 & 0.000 & 12.039 \\
\hline $2 \mathrm{H}-2,131$ & 10.23 & 0.628 & 0.379 & 1.705 & 11.932 & 2.462 & 10.606 & 0.568 & 2.273 & 1.894 & 7.954 & 1.894 & 0.568 & 0.000 & 0.000 & 17.803 \\
\hline $2 \mathrm{H}-2,141$ & 10.33 & 0.634 & 0.675 & 1.180 & 16.695 & 3.879 & 12.648 & 0.675 & 2.698 & 1.180 & 10.287 & 1.349 & 0.337 & 0.169 & 0.000 & 11.973 \\
\hline $2 \mathrm{H}-3,1$ & 10.43 & 0.641 & 0.534 & 1.957 & 12.989 & 3.203 & 8.541 & 0.534 & 3.203 & 2.313 & 9.075 & 1.246 & 0.712 & 0.178 & 0.178 & 16.726 \\
\hline $2 \mathrm{H}-3,11$ & 10.53 & 0.648 & 0.137 & 0.956 & 16.120 & 3.552 & 9.699 & 0.410 & 2.049 & 1.776 & 5.601 & 0.546 & 0.410 & 0.137 & 0.000 & 15.164 \\
\hline $2 \mathrm{H}-3,2 \mathrm{I}$ & 10.63 & 0.655 & 0.571 & 0.000 & 14.857 & 3.429 & 7.429 & 0.571 & 3.429 & 5.143 & 8.000 & 0.000 & 1.714 & 0.000 & 0.000 & 12.000 \\
\hline $2 \mathrm{H}-3,31$ & 10.73 & 0.662 & 1.185 & 3.317 & 22.986 & 2.133 & 7.109 & 1.185 & 3.081 & 1.422 & 3.555 & 0.948 & 0.711 & 0.474 & 0.000 & 12.322 \\
\hline $2 \mathrm{H}-3,4 \mathrm{I}$ & 10.83 & 0.669 & 0.229 & 6.179 & 16.476 & 0.000 & 8.009 & 1.602 & 1.831 & 1.144 & 5.263 & 0.458 & 1.373 & 0.000 & 0.000 & 15.103 \\
\hline $2 \mathrm{H}-3,5 \mathrm{I}$ & 10.93 & 0.676 & 0.699 & 0.233 & 11.422 & 1.865 & 5.128 & 0.932 & 2.331 & 0.699 & 7.925 & 2.331 & 0.233 & 0.233 & 0.000 & 18.415 \\
\hline $2 \mathrm{H}-3,61$ & 11.03 & 0.682 & 0.390 & 1.365 & 23.002 & 0.000 & 10.526 & 1.170 & 4.873 & 2.729 & 8.187 & 0.390 & 1.365 & 0.000 & 0.000 & 14.425 \\
\hline $2 \mathrm{H}-3.71$ & 11.13 & 0.687 & 0.617 & 0.772 & 31.944 & 1.080 & 10.803 & 0.309 & 2.469 & 0.463 & 6.327 & 0.309 & 0.000 & 0.000 & 0.000 & 10.648 \\
\hline $2 \mathrm{H}-3,81$ & 11.23 & 0.692 & 1.260 & 1.102 & 27.402 & 0.945 & 12.283 & 0.630 & 3.622 & 1.260 & 7.402 & 0.158 & 0.000 & 0.000 & 0.000 & 8.819 \\
\hline $2 \mathrm{H}-3,9 \mathrm{I}$ & 11.33 & 9.697 & 1.135 & 1.844 & 27.801 & 0.709 & 11.064 & 0.567 & 5.532 & 2.270 & 7.376 & 0.851 & 0.142 & 0.142 & 0.000 & 10.922 \\
\hline $2 \mathrm{H}-3,101$ & 11.43 & 0.703 & 0.649 & 1.297 & 21.790 & 1.686 & 7.523 & 1.167 & 2.464 & 1.297 & 5.966 & 0.649 & 0.259 & 1.297 & 0.000 & 15.564 \\
\hline $2 \mathrm{H}-3,1 \mathrm{H}$ & 11.53 & 0.708 & 1.923 & 1.224 & 23.951 & 1.224 & 7.343 & 0.525 & 2.448 & 2.797 & 6.119 & 1.224 & 0.175 & 2.622 & 0.000 & 10.140 \\
\hline $2 \mathrm{H}-3,12 \mathrm{I}$ & 11.63 & 0.714 & 0.978 & 1.075 & 30.596 & 0.195 & 10.068 & 1.564 & 2.150 & 0.978 & 8.700 & 0.782 & 0.000 & 0.195 & 0.000 & 8.993 \\
\hline $2 \mathrm{H}-3,13 \mathrm{I}$ & 11.73 & 0.719 & 1.125 & 0.643 & 26.367 & 1.125 & 10.289 & 0.482 & 4.341 & 1.125 & 6.109 & 0.482 & 0.161 & 1.125 & 0.000 & 10.289 \\
\hline $2 \mathrm{H}-3,14 \mathrm{I}$ & 11.83 & 0.723 & 2.817 & 0.939 & 23.474 & 1.643 & 7.512 & 0.469 & 3.286 & 0.939 & 7.277 & 0.469 & 0.235 & 2.113 & 0.235 & 8.685 \\
\hline $2 \mathrm{H}-4,1$ & 11.93 & 0.727 & 1.961 & 1.765 & 18.627 & 0.784 & 9.608 & 1.177 & 4.118 & 1.765 & 11.373 & 0.980 & 0.196 & 0.196 & 0.000 & 10.000 \\
\hline $2 \mathrm{H}-4,11$ & 12.03 & 0.731 & 2.179 & 0.871 & 34.858 & 0.218 & 8.932 & 2.179 & 2.614 & 0.871 & 3.922 & 0.218 & 0.218 & 0.000 & 0.000 & 10.893 \\
\hline $2 \mathrm{H}-4,2 \mathrm{I}$ & 12.13 & 0.734 & 1.013 & 2.703 & 22.973 & 0.000 & 13.514 & 2.196 & 4.730 & 0.845 & 8.784 & 0.845 & 0.676 & 0.000 & 0.000 & 10.304 \\
\hline $2 \mathrm{H}-4,3 \mathrm{I}$ & 12.23 & Ash D & & & & & & & & & & & & & & \\
\hline $2 \mathrm{H}-4,4 \mathrm{I}$ & 12.33 & Ash D & & & & & & & & & & & & & & \\
\hline $2 \mathrm{H}-4,51$ & 12.43 & 0.745 & 1.760 & 0.550 & 32.673 & 1.320 & 14.081 & 0.440 & 2.640 & 1.430 & 4.180 & 0.440 & 0.000 & 0.000 & 0.000 & 7.481 \\
\hline $2 \mathrm{H}-4,61$ & 12.53 & 0.748 & 1.560 & 1.404 & 30.889 & 1.248 & 13.729 & 0.780 & 2.652 & 2.184 & 5.148 & 0.624 & 0.312 & 0.936 & 0.000 & 7.488 \\
\hline $2 \mathrm{H}-4,71$ & 12.63 & 0.752 & 0.718 & 1.196 & 21.770 & 1.196 & 19.139 & 0.000 & 2.632 & 1.196 & 5.742 & 0.718 & 0.239 & 0.957 & 0.000 & 6.938 \\
\hline $2 \mathrm{H}-4,8 \mathrm{I}$ & 12.73 & 0.756 & 1.367 & 0.456 & 15.490 & 1.139 & 13.212 & 0.456 & 1.367 & 2.506 & 12.301 & 1.594 & 0.911 & 0.000 & 0.228 & 5.923 \\
\hline $2 \mathrm{H}-4,9 \mathrm{I}$ & 12.83 & 0.760 & 0.540 & 0.899 & 20.683 & 0.180 & 10.432 & 0.899 & 1.799 & 1.799 & 9.712 & 0.899 & 1.079 & 0.000 & 0.000 & 12.230 \\
\hline $2 \mathrm{H}-4,101$ & 12.93 & 0.765 & 0.641 & 2.350 & 14.744 & 0.000 & 11.111 & 1.496 & 1.496 & 1.496 & 9.829 & 1.923 & 1.068 & 0.427 & 0.000 & 8.333 \\
\hline $2 \mathrm{H}-4,111$ & 13.03 & 0.769 & 0.895 & 1.610 & 28.086 & 0.000 & 10.018 & 1.968 & 0.895 & 2.147 & 9.123 & 0.358 & 0.716 & 0.358 & 0.000 & 9.302 \\
\hline $2 \mathrm{H}-4,12 \mathrm{i}$ & 13.13 & 0.773 & 0.844 & 2.954 & 18.143 & 0.000 & 8.650 & 1.266 & 1.477 & 0.422 & 8.439 & 0.211 & 0.000 & 0.211 & 0.211 & 11.392 \\
\hline $2 \mathrm{H}-4,131$ & 13.23 & 0.777 & 0.500 & 0.250 & 16.750 & 0.000 & 8.250 & 1.500 & 1.250 & 1.250 & 7.750 & 1.000 & 0.000 & 0.500 & 0.000 & 16.250 \\
\hline $2 \mathrm{H}-4,14 \mathrm{I}$ & 13.33 & 0.782 & 0.484 & 0.969 & 16.465 & 0.242 & 8.959 & 2.421 & 2.421 & 1.695 & 7.990 & 0.000 & 0.000 & 0.000 & 0.000 & 10.170 \\
\hline $2 \mathrm{H}-5,1$ & 13.43 & 0.786 & 0.773 & 1.237 & 19.938 & 0.155 & 9.737 & 1.237 & 2.627 & 1.546 & 7.728 & 0.309 & 0.000 & 0.464 & 0.000 & 12.210 \\
\hline $2 \mathrm{H}-5,11$ & 13.53 & 0.790 & 0.220 & 0.440 & 28.634 & 0.000 & 9.692 & 1.542 & 1.101 & 0.220 & 7.930 & 0.440 & 0.440 & 0.440 & 0.220 & 7.709 \\
\hline $2 \mathrm{H}-5,2 \mathrm{I}$ & 13.63 & 0.795 & 0.000 & 1.444 & 20.397 & 0.542 & 4.874 & 1.083 & 0.722 & 0.902 & 8.484 & 0.902 & 0.181 & 1.625 & 0.181 & 17.274 \\
\hline $2 \mathrm{H}-5,3 \mathrm{I}$ & 13.73 & 0.799 & 0.733 & 0.293 & 19.501 & 1.026 & 7.478 & 1.906 & 1.026 & 1.026 & 8.211 & 0.147 & 0.147 & 1.466 & 0.000 & 13.343 \\
\hline $2 \mathrm{H}-5,41$ & 13.83 & 0.806 & 0.969 & 1.453 & 25.666 & 0.242 & 10.412 & 0.969 & 2.179 & 1.937 & 8.717 & 0.726 & 0.000 & 0.726 & 0.000 & 6.053 \\
\hline
\end{tabular}

FL appears to have deepened below the depth of Site 758 during the $\delta^{18} \mathrm{O}$ stages 20,18 , and 6 and during the glacial to interglacial transitions from $\delta^{18} \mathrm{O}$ stages 18 to 17,16 to 15,10 to 9,8 to 7,6 to 5 , and 2 to 1 .

The relationship among RSP\%, coarse fraction, and $\delta^{18} \mathrm{O}$ is generally characterized by low coarse fraction values and high RSP\% during interglacial stages and high coarse fraction values and low RSP\% during glacials (Fig. 3). The quasi-periodic 100k.y. cycles of the late Quaternary are superimposed on a long-term dissolution cycle. The mid-point of this cycle is between 400 and $550 \mathrm{ka}$, where dissolution is greatest as indicated by the highest values of RSP\% $(80 \%)$ and by the lowest coarse fraction values. The coarse fraction value of $1.89 \%$ at $510 \mathrm{ka}$ (Sample 121-758A$2 \mathrm{H}-1,101 \mathrm{~cm}$ ) is the lowest value of the past $2.9 \mathrm{Ma}$ (Farrell and Janecek, this volume). At the mid-point of the cycle, the increase in the abundances of the dissolution resistant species $P$. obliquiloculata, G. menardii, and G. tumida is mirrored by the decreased abundances of the dissolution susceptible species $G$. ruber, $G$. sacculifer, G. bulloides, and $G$. glutinata. The dissolution trend is clearly observed in the downcore record of $G$. tumida abundance (Fig. 2). At the beginning and end of the cycle, near 25 and $750 \mathrm{ka}$, enhanced $\mathrm{CaCO}_{3}$ preservation is indicated by high coarse fraction values and low RSP\%.

\section{DISCUSSION}

\section{Effects of Dissolution on Faunal Assemblages}

The relative abundance of planktonic foraminifers distributed on the seafloor is largely determined by the carbonate chemistry of the waters bathing the foraminifers. $\mathrm{CaCO}_{3}$ dissolution determines which species will be preserved in the fossil assemblage (Bé and Hutson, 1977; Cullen and Prell, 1984). We document variations in $\mathrm{CaCO}_{3}$ preservation based on faunal variations, faunal dissolution indices, and coarse fraction. The relationships between the coarse fraction values of each sample (a proxy for dissolution) and the abundances of eight major foraminifer species in the same sample are shown in Figure 5. The trends show that high coarse fraction is generally associated with high percentages of dissolution-susceptible species $G$. ruber, $G$. sacculifer, $G$. bulloides, and $G$. glutinata. Likewise, low coarse fraction is associated with high percentages of dissolution-resistant species $N$. dutertrei, G. menardii, $P$. obliquiloculata, and G. tumida. Good preservation is generally characterized by high abundances of the dissolution-susceptible species and high coarse fractions. Poor preservation is marked by high abundances of the dissolution-resistant species and low coarse fractions. The scatter about the trends is most likely related to a variety of ecological and/or non-preservational factors. Variations of $\% \mathrm{CaCO}_{3}$ at Site 758 primarily reflect dilution by terrigenous sediments and are therefore not a reliable index of dissolution (Farrell and Janecek, this volume).

The downcore pattern of dissolution occurs at a quasi-periodic cycle of $\sim 100 \mathrm{k} . \mathrm{y}$., closely linked to the glacial-interglacial climatic record interpreted from the $\delta^{18} \mathrm{O}$ to interglacials. These intervals are associated with the lowest RSP\% and the highest coarse fractions.

The atypical faunal composition observed between 490 and $550 \mathrm{ka}$ is attributed to a dramatic increase in $\mathrm{CaCO}_{3}$ dissolution 
Table 1 (continued).

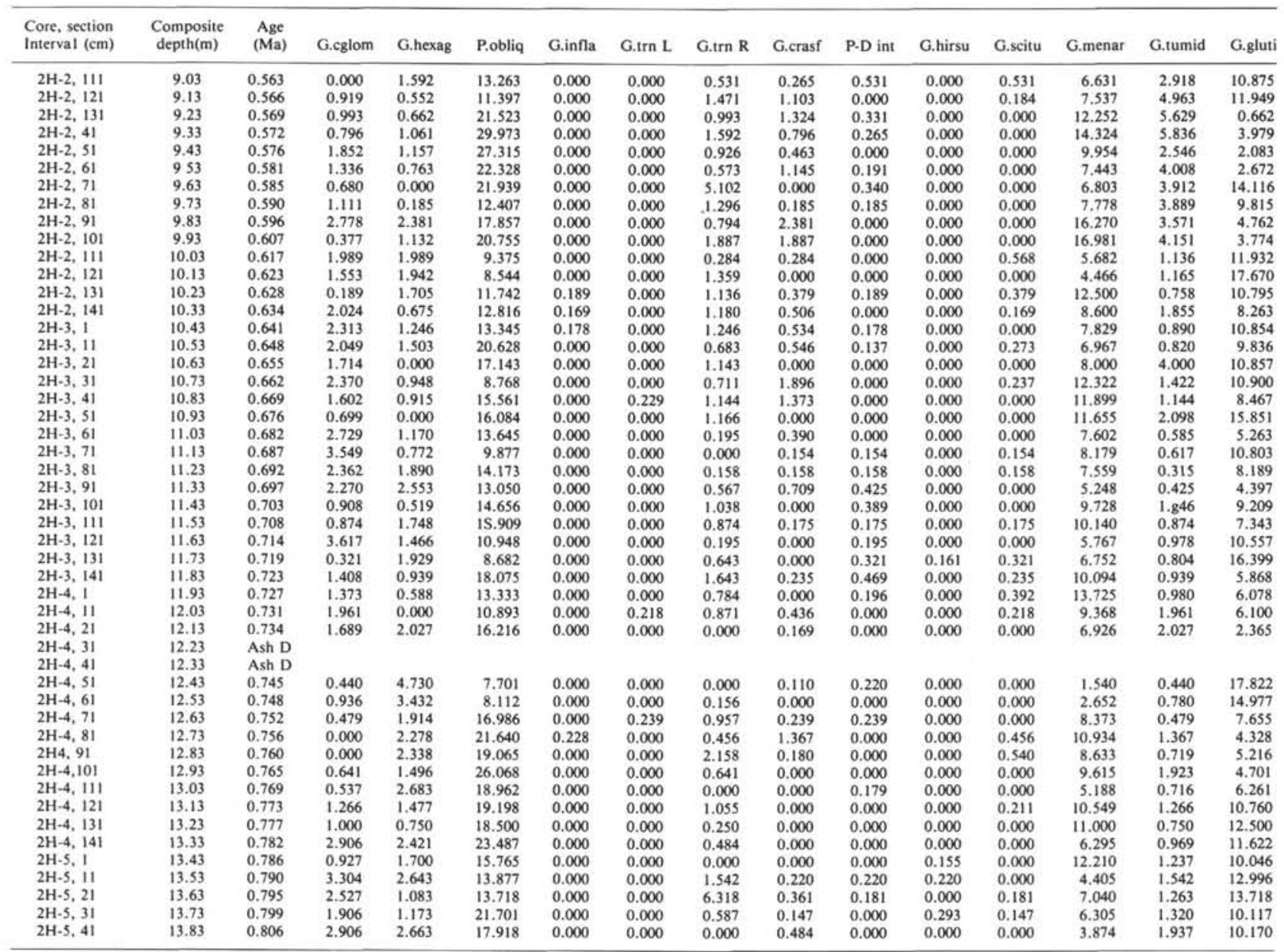

Table 2. Codes and descriptive statistics for planktonic foraminifer percentage data from Site 758 and from modern Indian Ocean sediments.

\begin{tabular}{|c|c|c|c|c|c|c|c|c|c|}
\hline \multirow[b]{2}{*}{ Foraminifer species } & \multirow[b]{2}{*}{ Code } & \multicolumn{4}{|c|}{$0-800 \mathrm{ka}$ at Site 758} & \multicolumn{4}{|c|}{ Modern surface sediments (Prell, 1985) } \\
\hline & & Average & St. Dev. & Minimum & Maximum & Average & St. Dev. & Minimum & Maximum \\
\hline Orbulina universa & O.unive & 0.8172 & 0.5673 & 0.0000 & 2.8169 & 0.5680 & 0.7550 & 0.0000 & 4.7035 \\
\hline Globigernoides conglobatus & G.cglob & 1.2957 & 0.9646 & 0.0000 & 6.1785 & 1.1826 & 1.4198 & 0.0000 & 8.1340 \\
\hline Globigerinoides ruber & Gruber & 18.3111 & 7.1216 & 3.1390 & 35.4883 & 16.7304 & 13.3172 & 0.0000 & 57.9832 \\
\hline Globigerinoides tenellus & G.tenel & 0.9507 & 1.0293 & 0.0000 & 4.7170 & 0.7683 & 1.1213 & 0.0000 & 7.7128 \\
\hline Globigerinoides sacculifer & G.saccu & 9.0541 & 3.1658 & 2.4000 & 19.1387 & 7.0198 & 5.8355 & 0.0000 & 24.7934 \\
\hline Sphaeroidinella dehiscens & S.dehis & 0.5667 & 0.5999 & 0.0000 & 2.4213 & 0.3052 & 0.6668 & 0.0000 & 5.6225 \\
\hline Globigerinella aequilateralis & G.aequi & 2.7479 & 1.1404 & 0.3559 & 5.9603 & 2.6528 & 2.1633 & 0.0000 & 9.1922 \\
\hline Globigerina calida & G.calid & 1.5496 & 0.7648 & 0.0000 & 5.1429 & 1.4470 & 1.4611 & 0.0000 & 9.1954 \\
\hline Globigerina bulloides & G.bullo & 6.2646 & 2.4325 & 0.5128 & 12.3007 & 14.7020 & 15.8717 & 0.2985 & 67.7711 \\
\hline Globigerina falconensis & G.falco & 0.4703 & 0.4771 & 0.0000 & 2.3310 & 1.6076 & 2.9509 & 0.0000 & 19.7183 \\
\hline Globigerina digitata & G.digit & 0.4159 & 0.4081 & $0.00 C 0$ & $1.851 \mathrm{~g}$ & 0.5673 & 1.1436 & 0.0000 & 12.7701 \\
\hline Globigerina rubescens & $\mathrm{G}$ rubes & 0.4113 & 0.5216 & 0.0000 & 2.6224 & 0.6422 & 1.0163 & 0.0000 & 5.2817 \\
\hline Globigerina pachyderma $\mathbf{R}$. & G.pac R & 0.1008 & 0.2258 & 0.0000 & 1.1472 & 2.0782 & 6.7909 & 0.0000 & 71.4286 \\
\hline Neogloboquadrina dutertrei & N.duter & 14.2684 & 3.9749 & 5.9226 & 26.1589 & 8.3539 & 6.9110 & 0.0000 & 35.1563 \\
\hline Globoquadrina conglormerata & G.cglom & 3.0841 & 2.0242 & 0.0000 & 9.3897 & 1.3604 & 2.2379 & 0.0000 & 12.9114 \\
\hline Globoquadrina hexagona & G.hexag & 1.3808 & 0.8873 & 0.0000 & 4.7305 & 1.0703 & 1.3389 & 0.0000 & 7.9625 \\
\hline Pulleniatina obliquiloculata & P.obliq & 14.7275 & 5.6887 & 5.1802 & 38.4615 & 3.6086 & 3.7212 & 0.0000 & 20.7792 \\
\hline Globorotalia inflata & G.infla & 0.0098 & 0.0484 & 0.0000 & 0.3968 & 7.1221 & 15.1551 & 0.0000 & 83.6420 \\
\hline Globorotalia truncatulinoides $\mathrm{L}$. & G.trn L & 0.0051 & 0.0339 & 0.0000 & 0.2392 & 1.2189 & 2.6638 & 0.0000 & 13.1313 \\
\hline Globorotalia truncatulinoides $\mathbf{R}$. & G.trn R & 0.3626 & 0.8224 & 0.0000 & 6.3177 & 0.4359 & 1.3529 & 0.0000 & 10.5802 \\
\hline Globorotalia crassaformis & G.crasf & 1.0398 & 1.0599 & 0.0000 & 5.5195 & 0.1480 & 0.4565 & 0.0000 & 4.3902 \\
\hline "P-D intergrade" & P-D.int & 0.7324 & 0.8934 & 0.0000 & 3.8136 & 1.6565 & 6.0487 & 0.0000 & 83.3333 \\
\hline Globorotalia hirsuta & G.hirsu & 0.1233 & 0.2557 & 0.0000 & 1.3393 & 0.2298 & 0.6540 & 0.0000 & 4.4369 \\
\hline Globorotalia scitula & G.scitu & 0.1310 & 0.1863 & 0.0000 & 0.7229 & 0.3476 & 0.5476 & 0.0000 & 4.6332 \\
\hline Globorotalia menardii & G.menar & 10.4066 & 4.3356 & 1.5402 & 26.9480 & 10.0006 & 12.3993 & 0.0000 & 65.8192 \\
\hline Globorotalia tumida & G.tumid & 2.0571 & 2.0668 & 0.0000 & 9.8655 & 1.2848 & 3.0309 & 0.0000 & 23.4657 \\
\hline Globigerinita glutinata & G.gluti & 8.7150 & 3.9905 & 0.6623 & 17.8218 & 12.8898 & 10.4878 & 0.0000 & 41.6667 \\
\hline
\end{tabular}




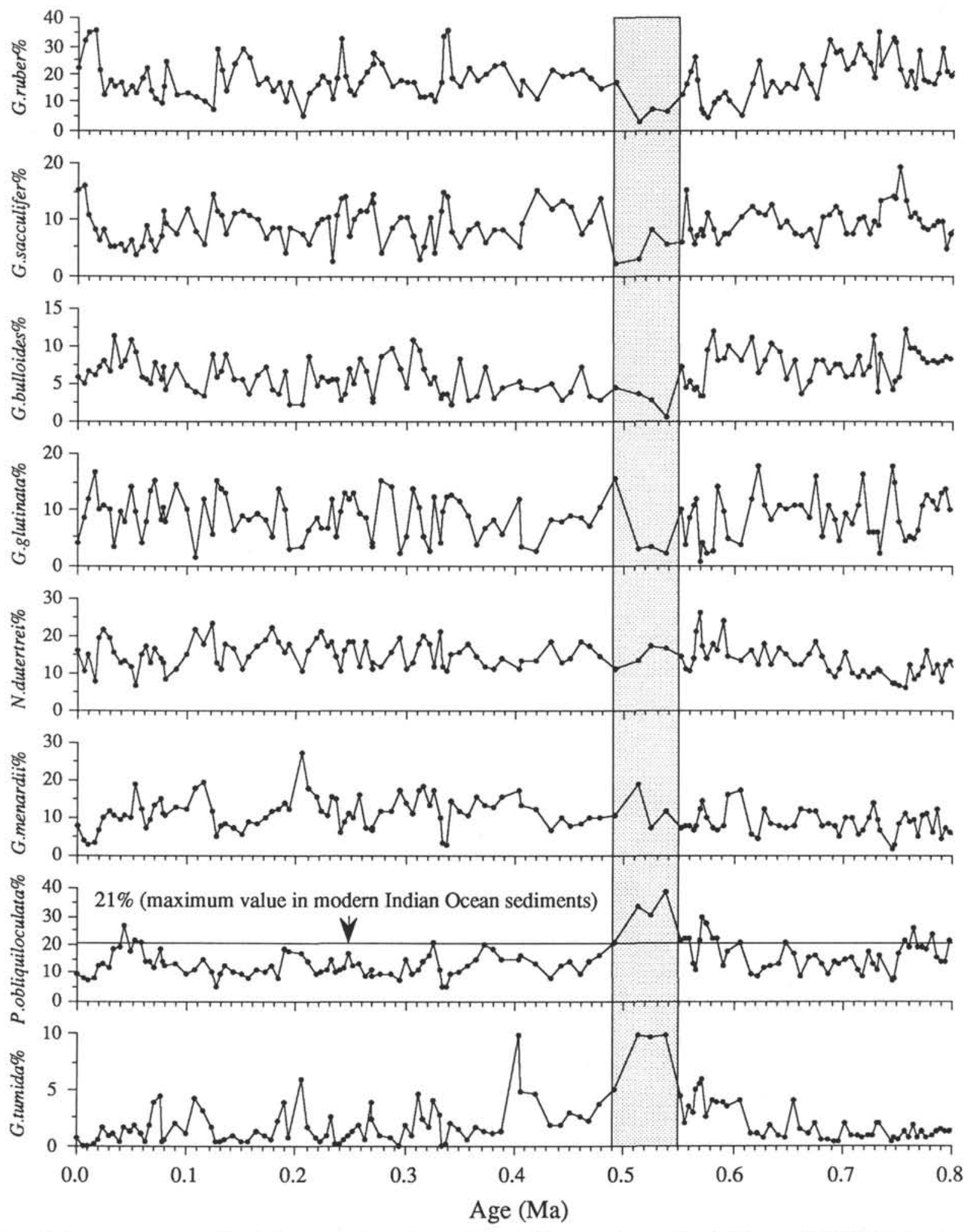

Figure 2. Percentage abundance of the eight major species of planktonic foraminifers plotted vs. age. The shaded region ( $490-550 \mathrm{ka})$ identifies a time of enhanced $\mathrm{CaCO}_{3}$ dissolution characterized by low abundances of G. ruber, G. sacculifer, G. bulloides, G. glutinata, and high abundances of $G$. menardii, $P$. obliquiloculata, G. tumida. The maximum value (21\%) of $P$. obliquiloculata observed in modern sediments from the Indian Ocean is marked by an arrow. 

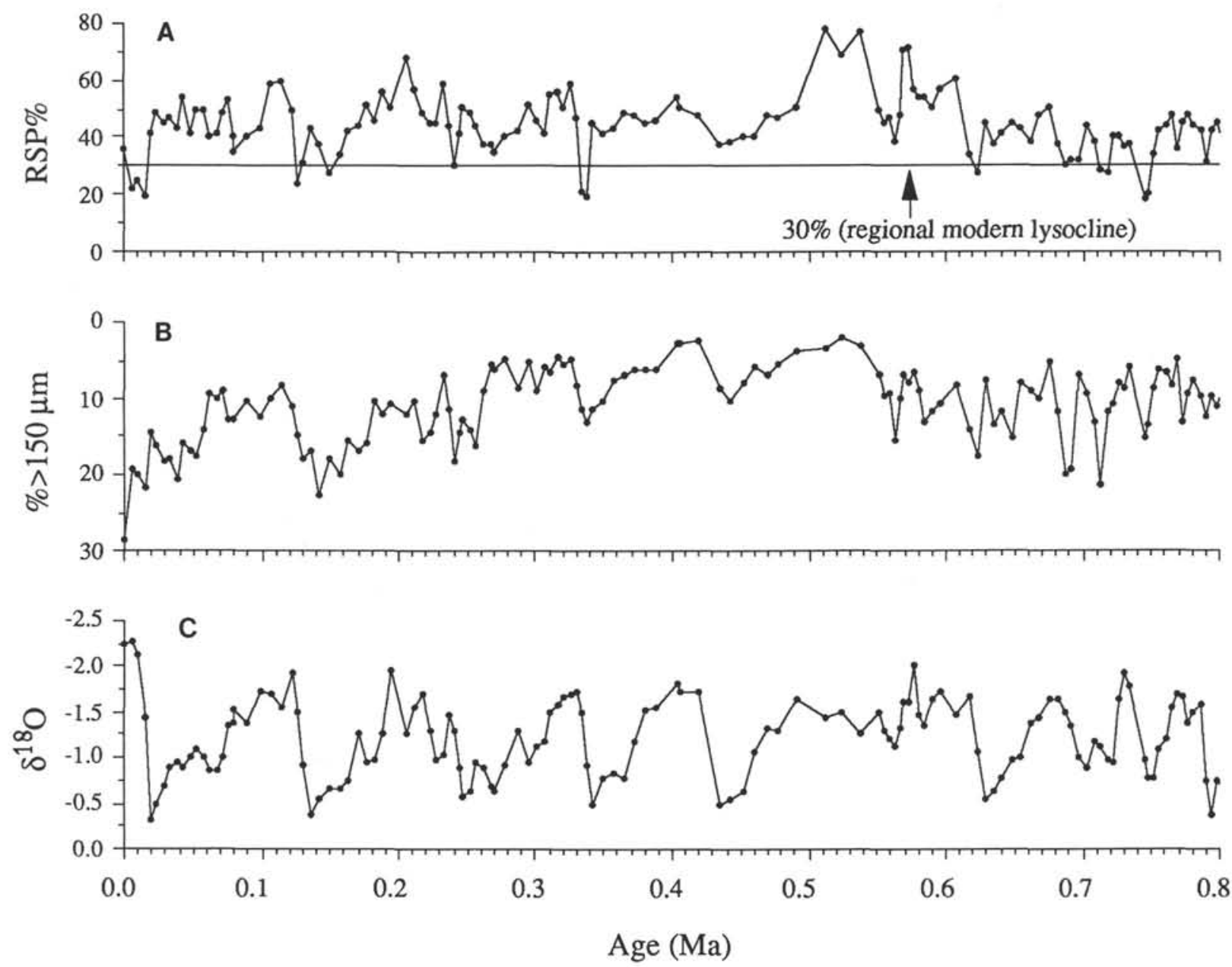

Figure 3. Percentage abundance of A. Resistant species ratio (RSP\%). Arrow shows the modern foraminifer lysocline as defined by the $30 \%$ RSP level (see text for explanation). B. Coarse fraction (wt $\%>150 \mu \mathrm{m})$, and C. $\delta^{18} \mathrm{O}(\% 0$ to PDB of $G$. sacculifer from the $300-355 \mu \mathrm{m}$ size range) from Site 758 plotted against age.

since species of similar susceptibility to dissolution show a common pattern. Between 490 and $550 \mathrm{ka}$, the relative percentages of susceptible species $G$. ruber, $G$. sacculifer, $G$. bulloides, and $G$. glutinata were significantly reduced while the abundances of the resistant species $P$. obliquiloculata, $G$. menardii, and $G$. tumida increased. This time interval is considered the mid-point of a long-term dissolution trend. The trend is also clearly observed in the RSP\%, the coarse fraction record, and in the downcore abundance record of G. tumida. The dissolution trend is the "Brunhes Dissolution Cycle" first described in sedimentary records from the equatorial Pacific (Adelseck, 1977). This cycle has been observed in other Indian Ocean records in water depths ranging from $533 \mathrm{~m}$ (Cullen and Droxler, 1990; Droxler et al., 1990) to over $4000 \mathrm{~m}$ (Peterson and Prell, 1985b). The cause of the cycle is unknown but has been related to forces both internal (Pisias and Rea, 1988) and external (Jansen et al., 1986) to the climate system.

\section{Ecological Factors Influencing Faunal Assemblages}

The relative abundance of planktonic foraminifers distributed on the seafloor is determined not only by dissolution, but also by the ecology of the overlying surface waters. Numerous studies have documented the great utility of planktonic foraminifers in paleoecological reconstructions. In sediments which have not been significantly altered by $\mathrm{CaCO}_{3}$ dissolution, variations in foraminifer faunal assemblages have been related to surface water conditions (Imbrie and Kipp, 1971; Cullen, 1984). The most important factors controlling foraminifer paleoecology include sea-surface temperature, salinity, and biological productivity.

As previously discussed, the SST in the Bay of Bengal is nearly constant on an annual basis. In addition, the SST during the last glacial maximum (LGM), 18,000 years ago, was nearly the same as it is today. Reconstructions indicate a cooling of SST at the LGM of no more than $1^{\circ} \mathrm{C}$ in this area (CLIMAP, 1976; 1981; Prell et al., 1980; Cullen, 1981). The low seasonality in modern SST, and the stable response of SST to glacial-interglacial cycles suggests that SST was probably not a major influence on faunal variation at Site 758 .

Past changes in the salinity gradient in the Bay of Bengal have been reconstructed along north-south transects based on planktonic foraminifer isotopes (Duplessy, 1982) and on faunal variations (Cullen, 1981). The faunal proxy of the salinity gradient was reconstructed along $90^{\circ} \mathrm{E}$ from $5^{\circ} \mathrm{S}$ to $20^{\circ} \mathrm{N}$ at three time intervals: the LGM; the middle of the transition (MT) from the LGM to the Holocene near $9 \mathrm{ka}$; and the Holocene (Cullen, 1981). During the LGM, the salinity gradient was weakened by an increase in salinity in the northern reaches of the Bay of Bengal. During the MT, the gradient was enhanced due to a freshening of 


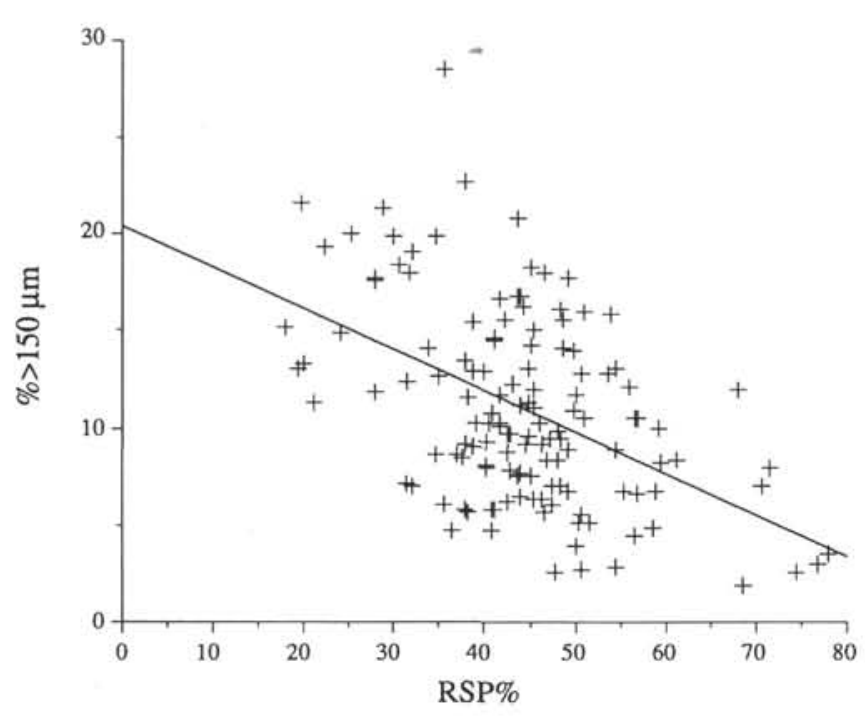

Figure 4. Scatter diagram of RSP\% vs. coarse fraction. Enhanced dissolution is characterized by high RSP\% values which are associated with low coarse fraction values.

waters in the north. In the Holocene, the salinity gradient was similar to that observed in the modern Bay of Bengal. The changes in the north were attributed to fluctuations in monsoonal precipitation and riverine input of fresh water (Cullen, 1981). The high salinity during the LGM was attributed to a reduction of monsoonal precipitation and riverine input. Likewise, the low salinity during the MT was attributed to an increase in precipitation and riverine input. Except for a brief period near the MT, the salinity in the southern Bay of Bengal $\left(\sim 5^{\circ} \mathrm{N}\right)$ has remained relatively stable at values between $33.5 \%$ and $34.0 \%$. Between the LGM and the MT, the salinity of the waters above Site 758 increased to about 34.5\% (Figure 20 in Cullen, 1981). This somewhat higher salinity in the central Bay of Bengal was attributed to an increase in the eastward advection of high salinity water carried by the Southwest Monsoon Current, which in turn was related to a stronger summer monsoon (Cullen, 1981). The largest change in salinity occurred in the northern part of the Bay of Bengal. Salinity at Site 758 was probably not directly affected by riverine input as the site is located far from the coasts. Changes in intensity of the Southwest Monsoon Current, however, may have had an effect on the salinity of the waters above Site 758. The degree to which the salinity may have changed over Site 758 and the ecological response of the foraminifers are discussed below.

If biological productivity at Site 758 changed significantly over the past $800 \mathrm{k} . \mathrm{y}$., it most likely changed in response to variations in the upwelling of nutrient-rich waters. Upwelling at Site 758 is presently minimal. The site is away from the upwelling regions associated with the equatorial divergence and with coastal upwelling zones in the Andaman Sea and off the coast of Sumatra. In Holocene and LGM sediments, variations in productivity related to upwelling intensity have been identified in the Andaman Sea and the northeastern Bay of Bengal (Fontugne and Duplessy, 1986). Greater organic carbon accumulation during the LGM was interpreted as indicating greater productivity and upwelling, which in turn was related to intensification of the Northeast monsoon. Only minor amounts of siliceous microfossils, which are considered an indication of upwelling and enhanced productivity, occur in the Quaternary sediments at Site 758 (Shipboard Scientific Party, 1989).

\section{Ecological Change at Site 758}

Having demonstrated that the late Quaternary faunal change at Site 758 is mainly controlled by the variation of $\mathrm{CaCO}_{3}$ dissolution, a dissolution-buffered strategy has to be used to extract the ecological signal from the foraminifer abundance data. To minimize the effects of dissolution, which would increase the relative abundance of dissolution-resistant species at the expense of the susceptible species, we followed the method of Cullen (1981) and recalculated the percentage of three dissolution-resistant species $N$. dutertrei, P. obliquiloculata, and G. menardii (Fig. 6). This method allows us to examine ecological variations in abundance of $N$. dutertrei, P. obliquiloculata, and G. menardii on a dissolution-buffered basis because these three species have a similar resistance to dissolution.

In the recalculated abundances we observe large variations $(10 \%-60 \%)$ in $N$. dutertrei which are superimposed on a longterm, but subtle increase of about $10 \%$ since at least $800 \mathrm{ka}$ (Fig. $6 \mathrm{~A})$. The high amplitude fluctuations, which occurred on a time scale of 10-50 k.y., were most common between 0 and $600 \mathrm{ka}$. We attribute these patterns to ecological factors which may reflect environmental change in the surface waters above Site 758. Two ecological factors have been associated with the abundance of $N$. dutertre $i$ in surface sediment samples. In the North Indian Ocean, high abundances of $N$. dutertrei appear closely linked to low sea-surface salinity (Cullen, 1981). Alternatively, high abundances of $N$. dutertrei in the eastern tropical Pacific (Berger, 1973) and in the Arabian Sea (Cullen, 1981) have been interpreted as indicating a shallow thermocline, a high nutrient level, and thus high productivity. To remove the dissolution imprint from the modern faunal abundance data (Prell, 1985) and to extract the ecologically controlled pattern of $N$. dutertrei, we recalculated the $N$. dutertrei percentage within three dissolution-resistant species $P$. obliquiloculata, G. menardii, and $N$. dutertrei (Fig. 7). This recalculation was identical to that performed on the downcore samples from Site 758. The core top values shown in Figure 7 are those in which the original sum of $P$. obliquiloculata, $G$. menardii, and $N$. dutertrei is greater than $30 \%$. This level was chosen to avoid core tops with low abundances of these three species. Low abundances result in relative proportions which are not statistically significant.

High abundances of $N$. dutertrei in core tops are observed beneath waters with lowest salinities $(<34 \% 0)$ in the northern Bay of Bengal, in the Andaman Sea, and off Sumatra Coast. Lowest abundances of $N$. dutertrei were observed in the Arabian Sea where salinity exceeds $36 \% 0$. Based on this observation, a simple interpretation of the increase in the abundance of $N$. dutertre $i$ with time is a long-term decrease in salinity at Site 758. Likewise, the large amplitude and high frequency fluctuations in the abundance of $N$. dutertrei may reflect relatively large and abrupt changes in salinity. Due to the great distance between Site 758 and the coast, it is unlikely that river run-off into the northern Bay of Bengal directly affected the salinity of the surface waters in the southern Bay of Bengal. A reduction in the salinity near Site 758 may have resulted from an increase in the advection of low salinity waters from the east through the Lombok Strait in response to a strengthening of the Southeast Asian Monsoon (Murray and Arief, 1988). Alternatively, there may have been a decrease in the advection of high salinity waters from the west by the Southwest Monsoon Current due to a weakening of the Southwest Monsoon. Finally, the salinity at Site 758 may have changed in response to regional fluctuations in the ratio of evaporation to precipitation.

We also observe a high concentration of $N$. dutertrei in core tops from regions where the thermocline shoals and upwelling of nutrient-rich water occurs. $N$. dutertrei is numerous in the central 


\section{Dissolution-susceptible species}
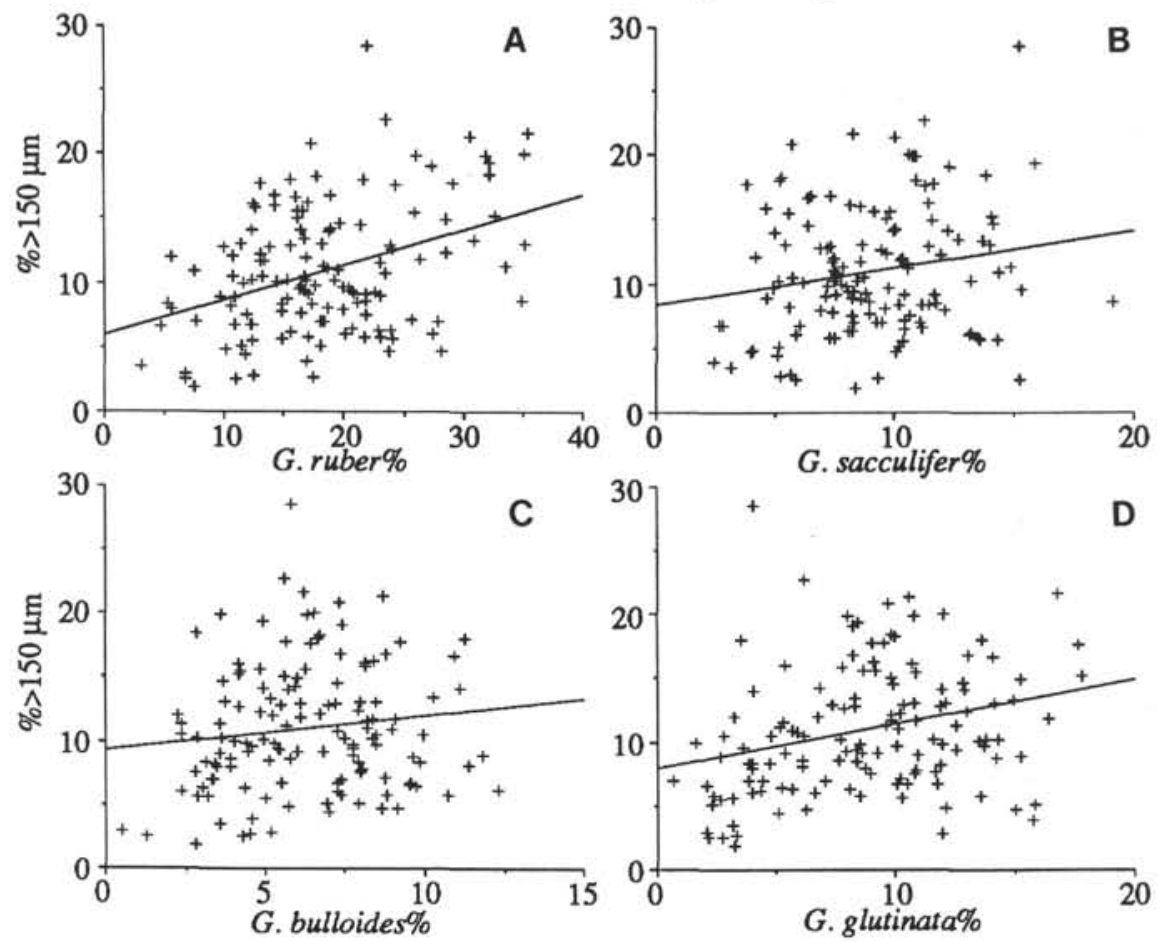

\section{Dissolution-resistant species}
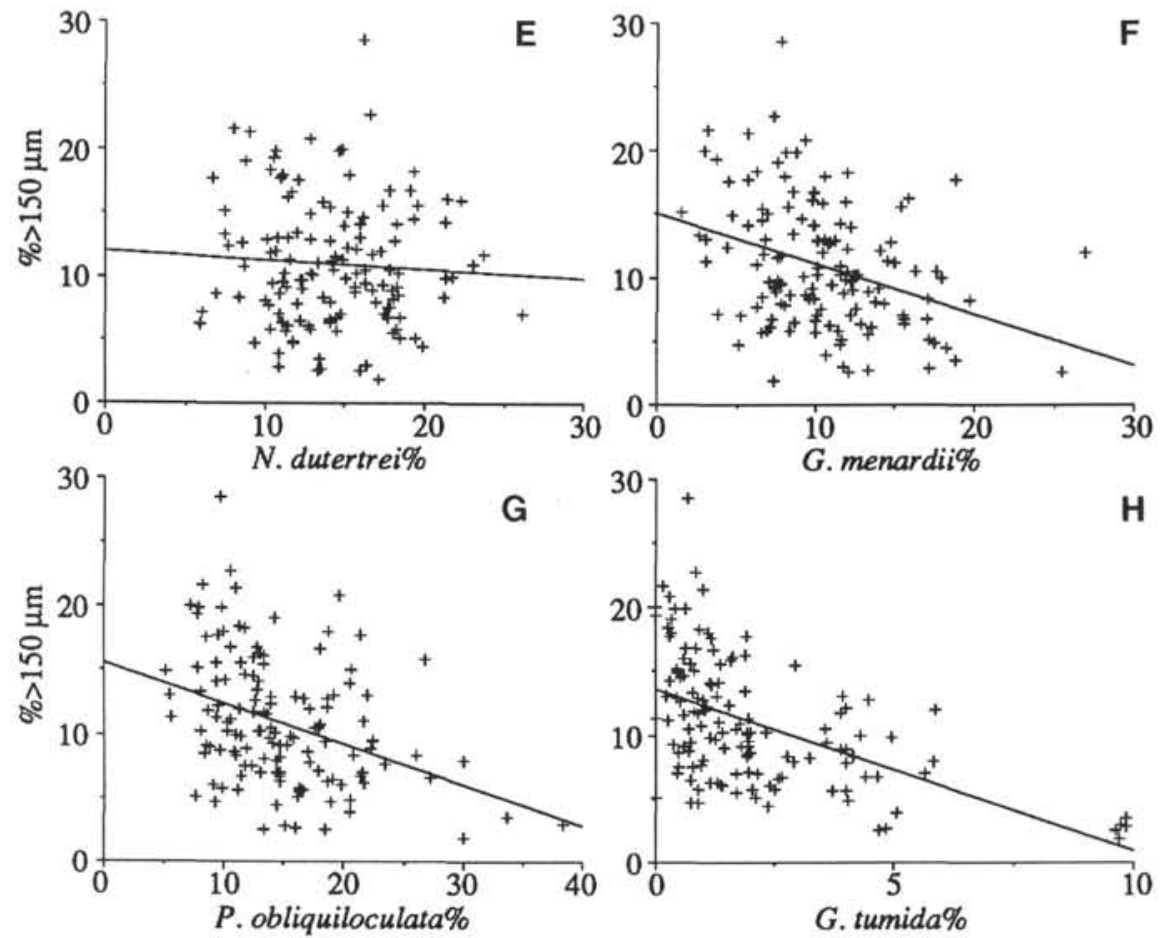

Figure 5. Scatter diagrams of coarse fraction vs. percentages of dissolution-susceptible species: A. G. ruber. B. G. sacculifer. C. G. bulloides. D. G. glutinata. Dissolution-resistant species: E. N. dutertrei. F. G. menardii. G. P. obliquiloculata. H. G. tumida. The lines are simple linear regressions. These plots show the relationships between foraminifer abundances and dissolution intensity. Strong dissolution is characterized by low coarse fraction and low percentages of dissolution-susceptible species (A-D). Enhanced preservation is characterized by high coarse fraction and low percentages of dissolution-resistant species $(\mathbf{E}-\mathbf{H})$. 

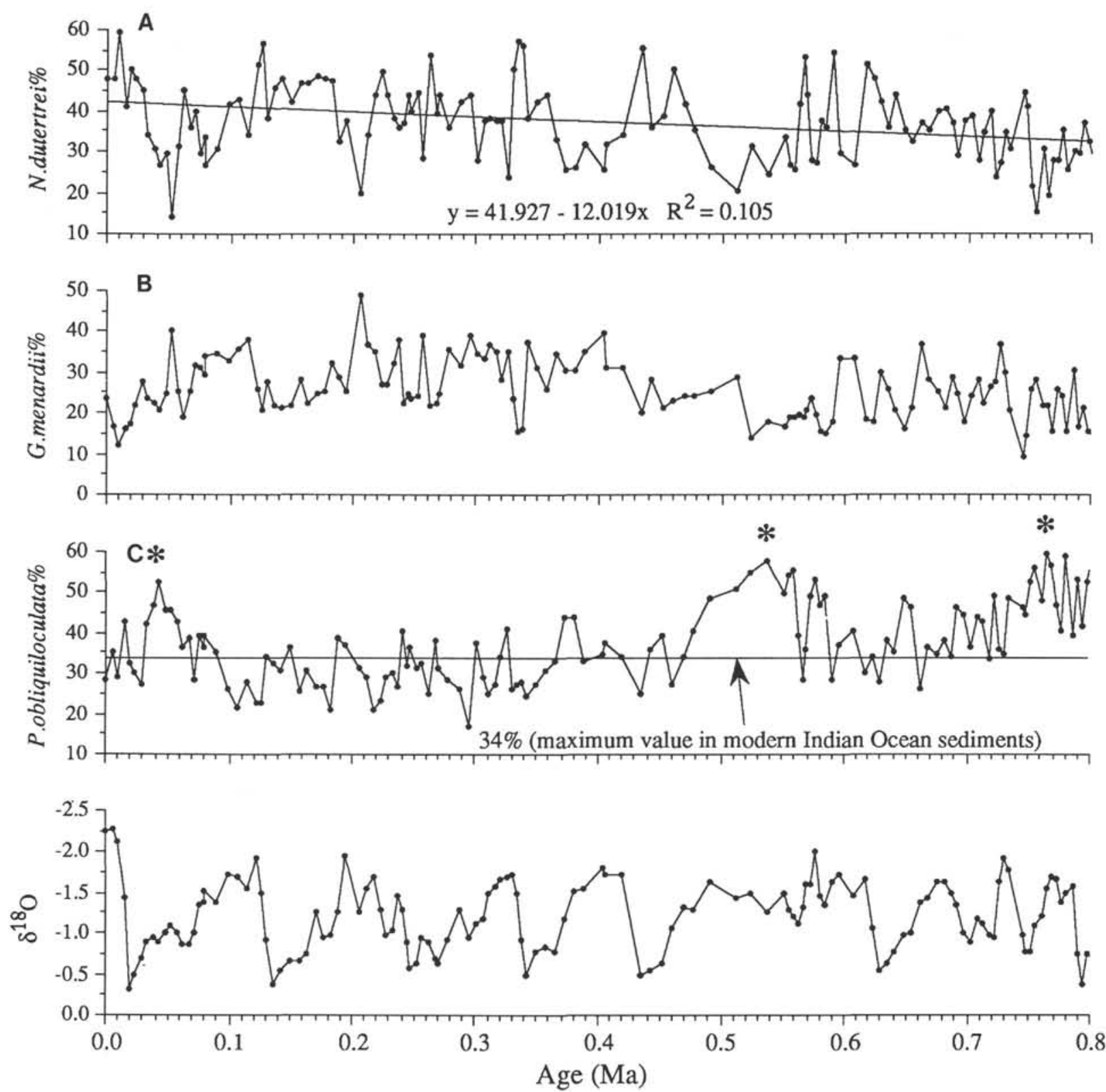

Figure 6. Recalculated percentage abundances of three dissolution-resistant species A. N. dutertrei. B. G. menardii. C. P. obliquiloculata plotted against age. The line in $\mathbf{A}$ is a simple linear regression which shows a $10 \%$ increase in $N$. dutertrei since $800 \mathrm{ka}$. For comparison to downcore values, the arrow in $\mathbf{C}$ shows the maximum surface sediment abundance (34\%) of $P$. obliquiloculata from Prell (1985) in terms of recalculated percentage abundances (see text for discussion). Samples at $43 \mathrm{ka}, 538 \mathrm{ka}$, and $765 \mathrm{ka}$ contain anomalously high $P$. obliquiloculata abundances and are marked with a "*".

and south tropical Indian Ocean underneath the North and South Equatorial Current where there is a strong equatorial divergence (Fig. 7). High concentrations of $N$. dutertrei are also observed in the regions of coastal upwelling in the Andaman Sea and Sumatra Coast (Fig. 7). The intensity of this coastal upwelling may be controlled by the strength of the Northeast Monsoon (Fontugne and Duplessy 1986). Based on this observation, changes in the abundance of $N$. dutertrei over time at Site 758 could be explained by fluctuations in productivity. These changes may be linked to the intensity of the Northeast Monsoon or the strength of the equatorial divergence.

A second intriguing ecological pattern observed in the Site 758 record is the anomalously high abundances of $P$. obliquiloculata. Several downcore samples from Site 758 have abundances of $P$. obliquiloculata that are much greater than the abundances ob- served in all (290) core top samples from the Indian Ocean (Prell, 1985). The downcore samples have greater abundances when calculated either as a percentage of all 27 species (Fig. 2) or recalculated as a percentage of the three dissolution resistant species ( $P$. obliquiloculata, G. menardii, and $N$. dutertrei) (Fig. $6 \mathrm{C}$ ). This indicates that $\mathrm{CaCO}_{3}$ preservation is not controlling the anomalously high abundances. For example, the highest abundance of $P$. obliquiloculata in core tops from the Indian Ocean occurs off the Sumatra Coast where values reach $21 \%$ (database from Prell, 1985) (Table 2). When recalculated as a percentage of only the dissolution resistant species, the $P$. obliquiloculata percentages in surface sediments are as high as $34 \%$. In the record from Site 758 , the percentage of $P$. obliquiloculata with respect to the entire fauna exceeds the surface sediment values during interglacial $\delta^{18} \mathrm{O}$ stages $3,13,14,15$, and 21 (Fig. 2). Likewise, 


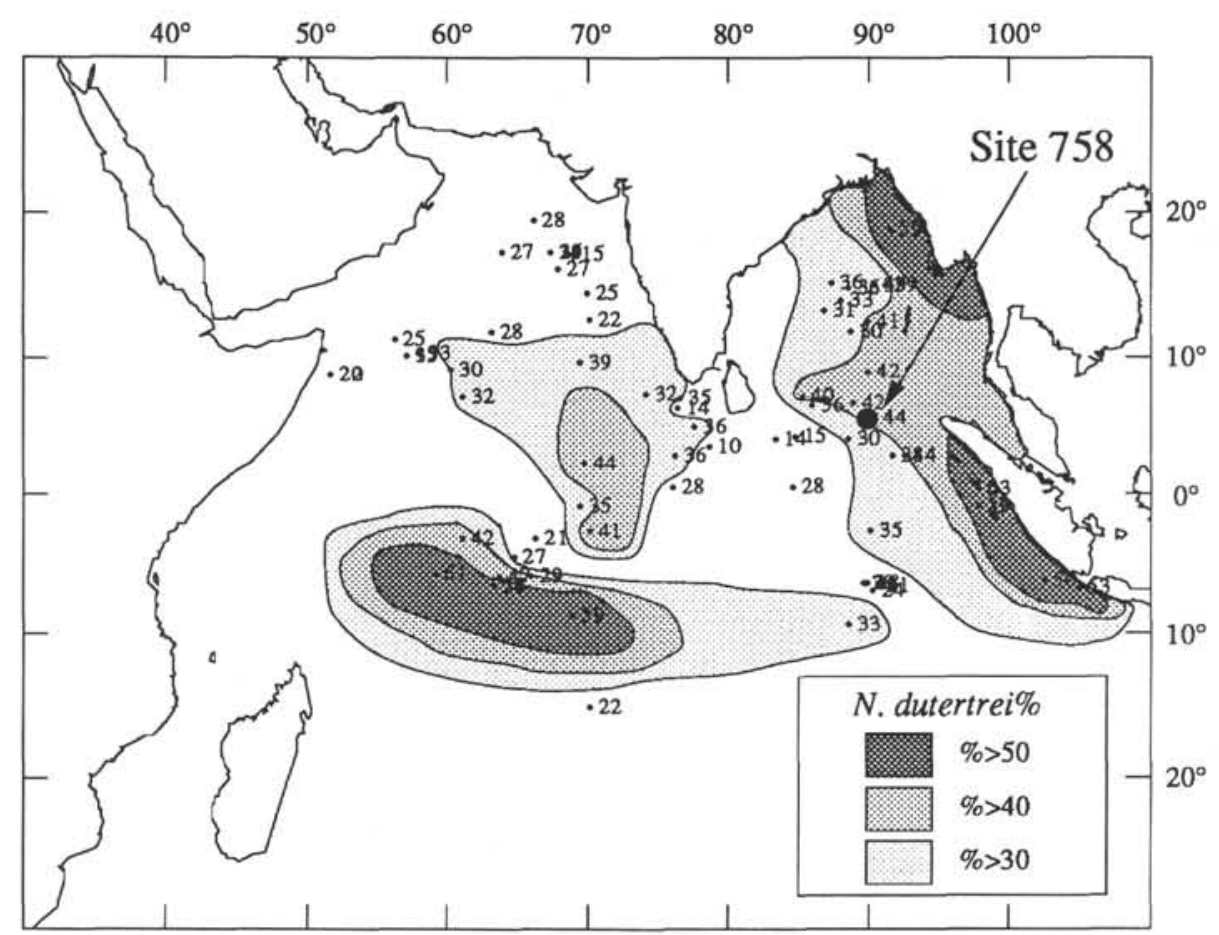

Figure 7. Spatial distribution of the recalculated abundance of $N$. dutertre $i$ in core top samples from the north Indian Ocean (database from Prell, 1985). High abundances are distributed in the Andaman Sea, off the Sumatra Coast, and beneath strong equatorial current systems of the tropical Indian Ocean.

when examined on the dissolution-buffered basis, the recalculated percentage of $P$. obliquiloculata abundances in Site 758 often exceeds the recalculated core top value of $34 \%$ (Fig. 6C). High abundances of $P$. obliquiloculata in downcore samples at Site 758 are, however, similar to the high abundances observed in core tops from the western tropical Pacific (Cullen and Prell, 1984).

We compared the faunal compositions of the core tops from the tropical Indo-Pacific with three representative downcore samples from Site 758 which have anomalously high abundances of $P$. obliquiloculata (samples from $\delta^{18} \mathrm{O}$ stage $3,14,21$ that are marked with an "*" in Fig. 6C). First, we recalculate the core top database (Prell, 1985) in terms of the three dissolution-resistant (P. obliquiloculata, G. menardii, and N. dutertrei) species. Second, we calculated the dissimilarity coefficient (using a squared chord distance) between the core top samples and the three downcore samples. In Figure 8, we observe a low dissimilarity between the core top samples from the western tropical Pacific and the downcore samples from Site 758 . We assume that similar faunas inhabit similar environments. Based on this assumption, the similarity of the core tops from the Pacific with the downcore samples suggests that surface water conditions in the modern western tropical Pacific were analogous to those in the northeastern Indian Ocean at certain times in the past. One possible explanation for the similar conditions during these times, is an increase in the advection of warm surface waters through the Indonesian Archipelago and into the northeastern Indian Ocean. This contention is supported by the observation of generally higher percentages of $P$. obliquiloculata during interglacials, when the throughflow between the tropical Indian and Pacific Oceans was probably greatest. During glacial times, low stands of the sea most likely restricted the exchange of water between these two regions. Alternatively, local oceanographic and climatic factors, such as the depth of the thermocline and the ratio of evaporation to precipitation, may have produced surface water conditions in the north- eastern Indian Ocean that were similar to those presently found in the western tropical Pacific.

\section{CONCLUSIONS}

1. Over the past 800 k.y., variations in the relative abundance of planktonic foraminifers at Site 758 were predominantly controlled by fluctuations in $\mathrm{CaCO}_{3}$ dissolution.

2. Dissolution varied at a cyclicity of $\sim 100$ k.y., in step with glacial/interglacial fluctuations. $\mathrm{CaCO}_{3}$ preservation was generally good during glacial intervals and poor during interglacials. Greatest preservation occurred on climate transitions from glacials to interglacials.

3. The $-100 \mathrm{k} . \mathrm{y}$. cycles are superimposed upon a long-term Brunhes Dissolution Cycle. The mid-point of this cycle, where dissolution was greatest, is centered between 400 and $550 \mathrm{ka}$ and is bounded by strong preservation at 25 and $750 \mathrm{ka}$.

4. Variations in the dissolution-buffered abundance of $N$. dutertre $i$ are attributed to ecological factors, most likely a decrease in sea-surface salinity or an increase in productivity.

5. The abundances of $P$. obliquiloculata in several downcore samples from Site 758 are greater than the abundances observed in core top samples from the Indian Ocean, but are similar to those found in core tops from the western tropical Pacific. This suggests that surface water conditions in the modern tropical Pacific were similar to conditions in the northeastern Indian Ocean at certain times in the past.

\section{ACKNOWLEDGMENTS}

The authors are grateful to A. Martin for assistance with planktonic foraminifer taxonomy and to W. Kang and T. Saha for help with sample preparation. We thank R. C. Thunell and T. Patterson for constructive reviews. Our work has greatly benefitted from discussions with J. L. Cullen, W. Howard, D. W. Murray, W. L. Prell, and Kuo-Yen Wei. This research was supported by 

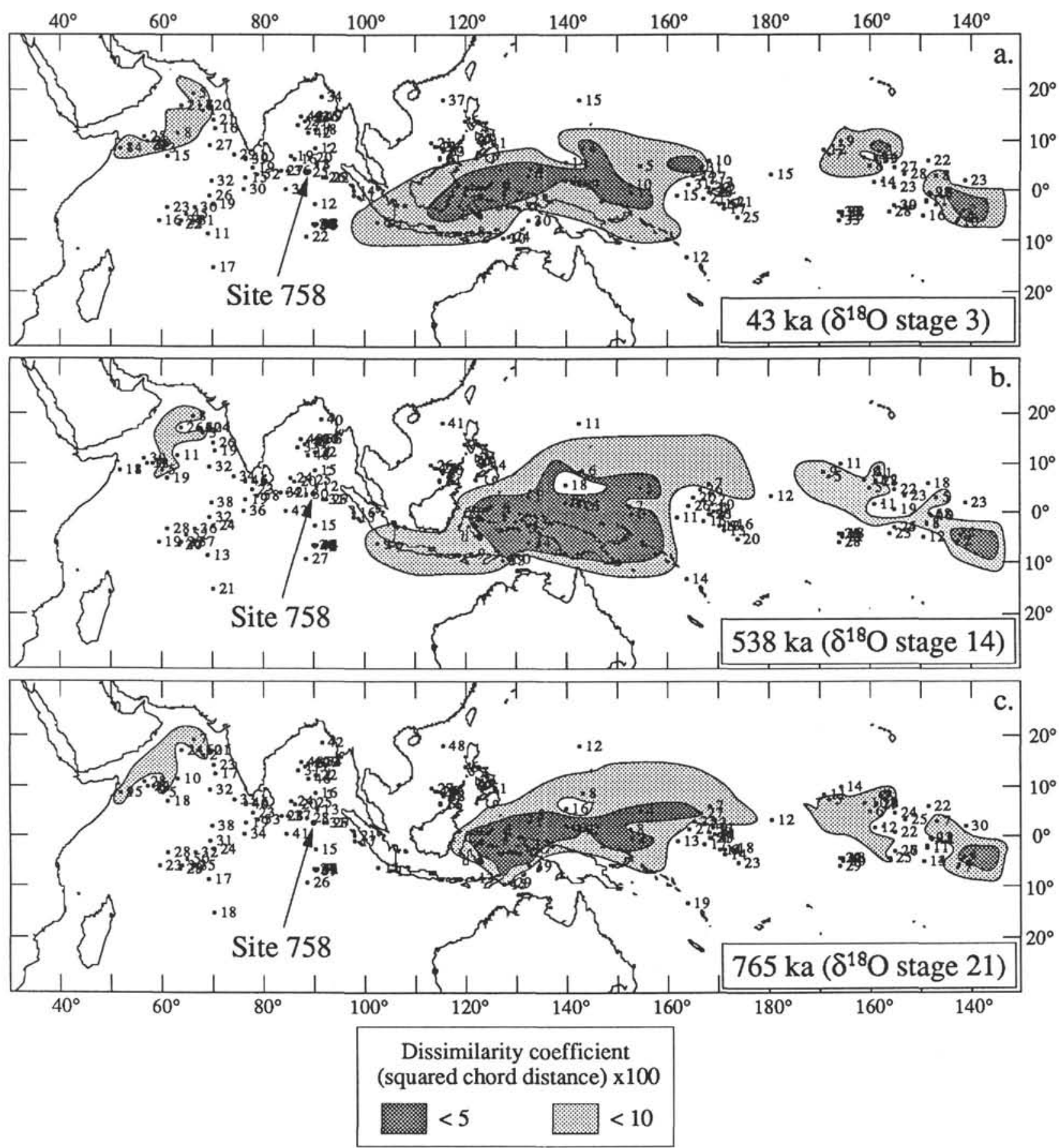

Figure 8. Spatial distribution of core top samples from the Indo-Pacific which contain foraminifer assemblages that are similar to the assemblages in three downcore samples (at $\sim 43 \mathrm{ka}, \sim 538 \mathrm{ka}$, and $\sim 765 \mathrm{ka}$ ) from Site 758. The core top samples which are most similar to the three downcore samples are from the tropical western Pacific and are considered modern analogues of the downcore samples from Site 758. The modern analogues are indicated by the lowest dissimilarity coefficients and are mapped as the shaded regions.

grants from JOI/USSAC (TAMRF-20244) (J. W. Farrell and T. Janecek), NSF/OCE 8911874 (D. W. Murray and J. W. Farrell), and a Brown University Teaching Assistantship (1989-1990) awarded to Min-Te Chen.

\section{REFERENCES}

Adelseck, C. G., Jr., 1977. Recent and late Pleistocene sediments from the eastern equatorial Pacific Ocean: sedimentation and dissolution. [Ph.D. dissert.], Univ. of California, San Diego.
1978. Dissolution of deep sea carbonate: preliminary calibration of preservational and morphological aspects. Deep-Sea Res., 24:1167-1185.

Bé, A.W.H., 1967. Foraminifera, families: Globigerinidae and Globorotaliidae, fiche No. 108. In Fraser, J. H. (Ed.), Fiches d'Identification $d u$ Zooplancton. Conseil Internat. l'Exploration Mer, Charlottenlund, Sheet 108.

Bé, A.W.H., and Hutson, W. H., 1977. Ecology of planktonic foraminifera and biogeographic patterns of life and fossil assemblages in the Indian Ocean. Micropaleontology, 27:369-414. 
Bé, A.W.H., and Tolderlund, D. S., 1971. Distribution and ecology of living planktonic foraminifera in surface waters of the Atlantic and Indian Oceans. In Funnel, B. M., and Riedel, W. R. (Eds.), The Micropaleontology of Oceans, Cambridge (Cambridge Univ. Press), 105-149.

Berger, W. H., 1968. Planktonic foraminifera: selective solution and paleoclimatic interpretation. Deep-Sea Res. Oceanogr. Abstr., 15: $31-43$.

1970. Planktonic foraminifera: selective solution and the lysocline. Mar. Geol., 8:111-138.

1973. Deep-sea carbonates: Pleistocene dissolution cycles. $J$. Foraminiferal Res., 3:187-195.

1975. Deep-sea carbonates: dissolution profiles from foraminiferal preservation. In Sliter, W. V., Bé, A.W.H., and Berger, W. H. (Eds.), Dissolution of Deep-Sea Carbonate, Spec. Publ. Found. Foraminiferal Res., 13:82-86.

1979. Preservation of foraminifera. In Lipps, J. H. (Ed.), Foraminifera Ecology and Paleoecology. SEPM Short Course, 6:105155.

Berger, W. H., Bonneau, M. -C., and Parker, F. L., 1982. Foraminifera on the deep-sea floor: lysocline and dissolution rate. Oceanol. Acta, $5: 249-258$.

CLIMAP project members, 1976. The surface of the ice-age Earth. Science, 191:1131-1137.

1981. Seasonal reconstructions of the Earth's surface at the last glacial maximum. Geol. Soc. Am., Map and Chart Ser., MC36:1-18.

Colborn, J. G., 1975. The thermal structure of the Indian Ocean. Internat. Indian Ocean Exped. Oceanogr. Monogr., 2:1-173.

Cullen, J. L., 1981. Microfossil evidence for changing salinity patterns in the Bay of Bengal over the last 20,000 years. Palaeogeogr., Palaeoclimatol., Palaeoecol., 35:315-356.

1984. Climatic variation in the northern Indian Ocean: analysis of the distribution, ecology, and preservation of planktonic foraminifera in late Quaternary sediments. [Ph.D. dissert.], Brown Univ., Providence.

Cullen, J. L., and Droxler, A. W., 1990. Late Quaternary variations in planktonic foraminiferal faunas and pteropod preservation in the equatorial Indian Ocean: In Duncan, R. A., Backman, J., Peterson, L. C., et al., Proc. ODP, Sci. Results, 115: College Station, TX (Ocean Drilling Program), 579-588.

Cullen, J. L., and Prell, W. L., 1984. Planktonic foraminifera of the northern Indian Ocean: distribution and preservation in surface sediments. Mar. Micropaleontol., 9:1-52.

Curray, J. R., and Moore, D. G., 1971. Growth of the Bengal deep-sea fan and denudation in the Himalayas. Geol. Soc. Am. Bull., 82:563-572.

Droxler, A. W., Haddad, G. A., Mucciarone, D. A., and Cullen, J. L., 1990. Pliocene-Pleistocene aragonite cyclic variations in Holes 714A and 716B (The Maldives) compared with Hole 633A (the Bahamas): Records of climate-induced $\mathrm{CaCO}_{3}$ preservation at intermediatewater depths. In Duncan, R. A., Backman, J., Peterson, L. C., et al., Proc. ODP, Sci. Results, 115: College Station, TX (Ocean Drilling Program), 539-577.

Duplessy, J.-C., 1982. Glacial to interglacial contrasts in the northern Indian Ocean. Nature, 295:494-498.

Fairbanks, R. G., Sverdlove, M., Free, R., Wiebe, P. H., and Bé, A.W.H., 1982. Vertical distribution and isotopic fractionation of living planktonic foraminifera from the Panama Basin. Nature, 298:841-844.

Fontugne, M. R., and Duplessy, J. -C., 1986. Variations of the monsoon regime during the upper Quaternary: evidence from carbon isotopic record of organic matter in North Indian Ocean sediment cores. Palaeogeogr., Palaeoclimatol., Palaeoecol., 56:69-88.

Goldberg, E. D., and Griffin, J. J., 1970. The sediments of the northern Indian Ocean. Deep-Sea Res. Oceanogr. Abstr. 17:513-537.

Hutson, W. H., and Prell, W. L., 1980. A paleoecological transfer function, FI-2, for Indian Ocean planktonic foraminifera. J. Paleontol., 54:381-399.

Imbrie, J., Hays, J. D., Martinson, D. G., McIntyre, A., Mix, A. C., Morley, J. J., Pisias, N. G., Prell, W. L., and Shackleton, N. J., 1984. The orbital theory of Pleistocene climate: support from a revised chronology of the marine $\delta^{18} \mathrm{O}$ record. In Berger, A., Imbrie, J., Hays,
H., Kukla, G., and Saltzman, B. (Eds.), Milankovitch and Climate, Part I, Dordrecht (Reidel), 269-305.

Imbrie, J. and Kipp, N. G., 1971. A new micropaleontological method for quantitative paleoclimatology: application to a late Pleistocene Caribbean core. In Turekian, K. K. (Ed.), The Late Cenozoic Glacial Ages, New Haven (Yale Univ. Press), 71-181.

Jansen, J.H.F., Kuijpers, A., Troelstra, S. R., 1986. A mid-Brunhes climatic event: long-term changes in global atmospheric and ocean circulation. Science, 232: 619-622.

Kipp, N. G., 1976. New transfer function for estimating past sea-surface conditions from sea-bed distribution of planktonic foraminiferal assemblages in the North Atlantic. Mem. Geol. Soc. Am., 145:3-41.

Malmgren, B. A., 1983. Ranking of dissolution susceptibility of planktonic foraminifera at high latitudes of the south Atlantic Ocean. Mar. Micropaleontol., 8:183-191.

Murray, S. P., and Arief, D., 1988. Throughflow into the Indian Ocean through the Lombok Strait, January 1985-January 1986. Nature, 333:444-447.

Parker, F. L., 1962. Planktonic foraminiferal species in Pacific sediments. Micropaleontology, 8:219-254.

Parker, F. L., and Berger, W. H., 1971. Faunal and solution patterns of planktonic foraminifera in surface sediments of the South Pacific. Deep-Sea Res. Oceanogr. Abstr., 18:73-107.

Peterson, L. C., and Prell, W. L., 1985a. Carbonate dissolution in recent sediments of the eastern equatorial Indian Ocean: preservation patterns and carbonate loss above the lysocline. Mar. Geol., 64:259-290. 1985b. Carbonate preservation and rates of climatic change: an $800 \mathrm{kyr}$ record from the Indian Ocean. In Sundquist E. T., and Broecker, W. S. (Eds.), The Carbon Cycle and Atmospheric $\mathrm{CO}_{2}$ : Natural Variations Archean to Present, Washington (Am. Geophys. Union), 32:251-270.

Pisias, N. G., and Rea, D. K., 1988. Late Pleistocene paleoclimatology of the central equatorial Pacific: sea-surface response to the southeast tradewinds. Paleoceanography, 3:21-37.

Prell, W. L., 1984. Variation of monsoon upwelling: a response to changing solar radiation. In Hansen J. E., and Takahashi, T. (Eds.), Climate Process and Climate Sensitivity, Am. Geophys. Union, Maurice Ewing Ser., 29:48-57.

1985. The stability of low-latitude sea-surface temperature: an evaluation of the CLIMAP reconstruction with emphasis on the positive SST anomalies. Dept. Energy Tech. Rept., TR-025.

Prell, W. L. and Curry, W. B., 1981. Faunal and isotopic indices of monsoonal upwelling: western Arabian Sea. Oceanol. Acta, 4:91-98.

Prell, W. L., Hutson, W. H., Williams, D. F., Bé, A.W.H., Geitzenauer, R., and Molfino, B., 1980. Surface circulation of the Indian Ocean during the last glacial maximum, approximately $18,000 \mathrm{yr}$ B.P., Quat. Res., 14:309-336.

Prell, W. L., Imbrie, J., Martinson, D. G., Morley, J. J., Pisias, N. G., Shackleton, N. J., and Streeter, H. F., 1986. Graphic correlation of oxygen isotope stratigraphy: application to the late Quaternary. $\mathrm{Pa}$ leoceanography, 1:137-162.

Prell, W. L., Marvil, R. E., and Luther, M. E., 1990. Variability in upwelling fields in the northwestern Indian Ocean 2. data-model comparison at 9000 years B.P. Paleoceanography, 5:447-457.

Ravelo, A. C., Fairbanks, R. G., and Philander, G., 1990. Reconstructing tropical Atlantic hydrography using planktonic foraminifera and an ocean model. Paleoceanography, 5:409-431.

Rodolfo, K. S., 1967. Sediments of the Andaman Sea, northeastern Indian Ocean. Mar. Geol., 7:371-402.

Shackleton, N. J., and Opdyke, N. D., 1973. Oxygen isotope and paleomagnetic stratigraphy of equatorial Pacific core V28-238: oxygen isotope temperature and ice volumes on a 105 and 106 year scale. Quat. Res., 3:39-55.

1976. Oxygen isotope and paleomagnetic stratigraphy of core V28-239, late Pliocene to latest Pleistocene. In Cline, R. M., and Hays, J. D. (Eds.), Investigations of Late Quaternary Paleoceanography and Paleoclimatology, Mem. Geol. Soc. Am., 145:449-464.

Shipboard Scientific Party, 1989. Site 758. In Peirce, J., Weissel, J., et al., Proc. ODP, Init. Repts. 121: College Station, TX (Ocean Drilling Program), 359-453. 
Thompson, P. R., Bé, A.W.H., Duplessy, J. C., and Shackleton, N. J., 1979. Disappearance of pink-pigmented Globigerinoides ruber at $120,000 \mathrm{yr}$ B.P. in the Indian and Pacific Oceans. Nature, 280:554 558.

Thunell, R. C., and Honjo, S., 1981a. Planktonic foraminiferal flux to the deep ocean: sediment trap results from the tropical Atlantic and the central Pacific. Mar. Geol., 40:237-253.

$1981 \mathrm{~b}$. Calcite dissolution and the modification of planktonic foraminiferal assemblages. Mar. Micropaleontol., 6:169-182.

Thunell, R. C., and Reynolds, L. A., 1984. Sedimentation of planktonic foraminifera: seasonal changes in species flux in the Panama Basin. Micropaleontology, 30:243-262.
Wyrtki, K., 1973. Physical oceanography of the Indian Ocean. In Zeitzschel, B., and Gerlach, S. A. (Eds.), The Biology of the Indian Ocean, Vol 3: New York (Springer-Verlag), 18-36.

1988. Oceanography Atlas of the International Indian Ocean Expedition, New Delhi (Amerind).

\section{Ms 121B-174}

Date of initial receipt: 17 April 1990

Date of acceptance: 25 November 1990 